\title{
The long path of human placenta, and its derivatives, in regenerative medicine
}

\author{
Antonietta R. Silini, Anna Cargnoni, Marta Magatti, Stefano Pianta and Ornella Parolini* \\ Centro di Ricerca "E. Menni", Fondazione Poliambulanza Istituto Ospedaliero, Brescia, Italy
}

In the 1800s, a baby born with a caul, a remnant of the amniotic sack or fetal membranes, was thought to be lucky, special, or protected. Over time, fetal membranes lost their legendary power and were soon considered nothing more than biological waste after birth. However, placenta tissues have reclaimed their potential and since the early 1900s an increasing body of evidence has shown that these tissues have clinical benefits in a wide range of wound repair and surgical applications. Nowadays, there is a concerted effort

OPEN ACCESS

Edited by:

Martijn Van Griensven, Technical University Munich,

Germany

Reviewed by:

Vasif Nejat Hasirci,

Middle East Technical University,

Turkey

Susanne Wolbank

Ludwig Boltzmann Institute for

Clinical and Experimental

Traumatology, Austria

*Correspondence:

Ornella Parolini

ornella.parolini@poliambulanza.it

Specialty section: This article was submitted to Tissue

Engineering and Regenerative

Medicine, a section of the journal

Frontiers in Bioengineering and

Biotechnology

Received: 27 July 2015

Accepted: 28 September 2015

Published: 19 October 2015

Citation:

Silini AR, Cargnoni A, Magatti M,

Pianta S and Parolini O (2015) The long path of human placenta, and its derivatives, in regenerative medicine.

Front. Bioeng. Biotechnol. 3:162.

doi: 10.3389/fbioe.2015.00162 to understand the mechanisms underlying the beneficial effects of placental tissues, and, more recently, cells derived thereof. This review will summarize the historical and current clinical applications of human placental tissues, and cells isolated from these tissues, and discuss some mechanisms thought to be responsible for the therapeutic effects observed after tissue and/or cell transplantation.

Keywords: human term placenta, amniotic and chorionic membranes, umbilical cord, stem cells, clinical trials, immunomodulation, paracrine effect, regenerative medicine

\section{A SHORT HISTORY OF THE CLINICAL USES OF HUMAN TERM PLACENTA}

Human placenta has been traditionally used in Chinese medicine for centuries. The Compendium of Materia Medica was published in 1593 by one of the first and greatest biologists and pharmaceutical experts of China, Li Shi-Zhen (Figure 1). This medical text is a Chinese record of substances with medical properties, and it contains a section entirely devoted to the medical uses of human placenta "zi he chi" as a medicine (Young and Benyshek, 2010). At that time, eating the placenta was thought to be beneficial but since then there has been a shift of paradigms in which scientific rationale supports clinical benefit of placental tissues, or derivatives, for treating patients afflicted by a variety of diseases. As discussed below, the earliest reported applications of the placenta (after Li Shi-Zhen's) were focused on fetal membranes. The first reports showing that the placenta also harbors cells, which could have stem/progenitor properties, ultimately giving rise to their potential use in regenerative medicine, were published many years later (Figure 1), (Bailo et al., 2004; Fukuchi et al., 2004; Igura et al., 2004; In 't Anker et al., 2004; Soncini et al., 2007; Troyer and Weiss, 2008).

The first documented use of fetal membranes as a surgical material in skin transplantation came more than 3 decades after the placenta was initially reported to possess medicinal properties. In 1910, Davis (1910) showed that the use of amniotic membrane (AM) in skin grafting gave superior results when compared to xenograft or cadaveric coverings. Shortly afterwards in 1913, Stern (1913) and Sabella (1913) reported the use of the AM for treating skin wounds. They applied intact amniotic tissues to skin burns and ulcers and then covered them with dressings. Upon removal of the dressings 2 days 


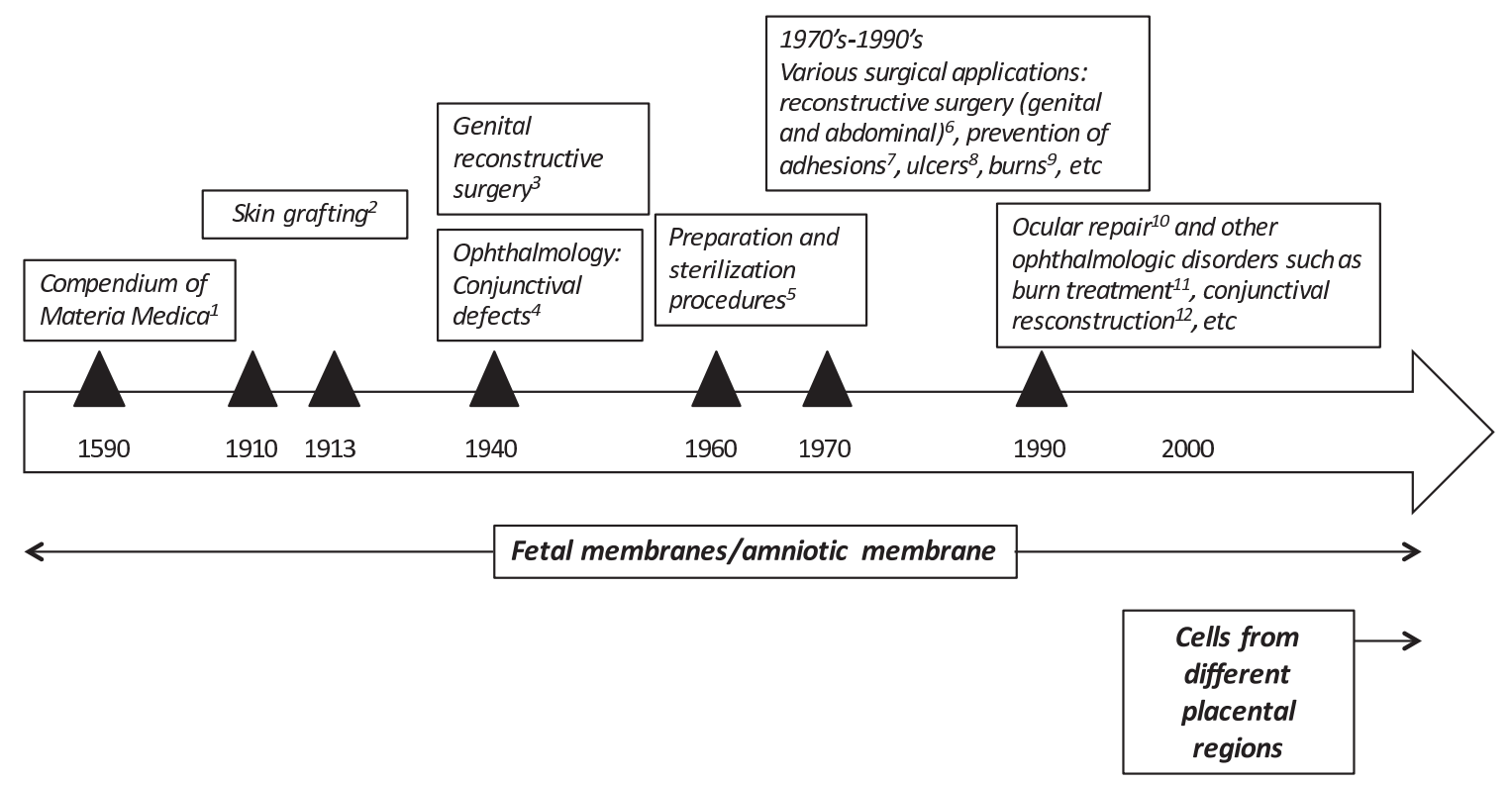

FIGURE 1 | Historical uses of human term placental tissues and cells derived thereof. ${ }^{1}$ Chinese medical text published in 1593 by Li Shi-Zhen; ${ }^{2}$ Davis (1910) and Stern (1913) and Sabella (1913); ${ }^{3}$ Brindeau (1934) and Burger (1937); ' De Rotth (1940), Sorsby and Symons (1946); Sorsby et al. (1947); 5 Dino et al. (1965); ${ }^{6}$ Trelford-Sauder et al. (1977); Trelford-Sauder et al. (1978), Silverton et al. (1979); Dhall (1984), Nisolle and Donnez (1992), Georgy and Aziz (1996), Gharib et al. (1996); ${ }^{7}$ Trelford-Sauder et al. (1978); Muralidharan et al. (1991), Young et al. (1991); Arora et al. (1994), Rennekampff et al. (1994); ${ }^{8}$ Troensagaard-Hansen (1950); Bennett et al. (1980), Subrahmanyam (1995); ${ }^{9}$ Gruss and Jirsch (1978) and Bose (1979); ${ }^{10}$ Lee and Tseng (1997); Tseng et al. (1997), ${ }^{11,12}$ reviewed in Fetterolf and Snyder (2012).

later, the authors reported that the amnion had integrated with the patient's tissues. They also reported lack of infection, a significant decrease in pain, and an increased rate of re-epithelialization of the traumatized skin surface in patients treated with amnion.

More than 20 years passed before another study would report the use of amniotic tissues for wound repair or surgery. Around 1940, the AM was increasingly being used successfully in different applications. In the late 1930s, Brindeau (1934) and Burger (1937) reported the successful use of amnion for vaginal reconstruction surgery in a patient with Mullerian agenesis. Shortly afterwards, the AM was first applied in ophthalmology to repair conjunctival defects (De Rotth, 1940) and burns (Sorsby and Symons, 1946; Sorsby et al., 1947). Ophthalmology would later go on to be one of the most popular applications of the AM to date.

Following these and other studies, between 1940 and 1970, a number of clinical trials were published reconfirming the successful use of the AM for skin injuries. In 1940, the first paper was published describing the use of amnion for the prevention of meningocerebral adhesions following head injury. The authors reported lack of adhesions and lack of rejection 60 days after surgery, and "disappearance" of the amnion after 30 days (Chao et al., 1940). Later that decade, and following Burger's work on vaginal reconstruction, Kubanyi (1947) used amnion in patients undergoing abdominal surgery with an enterocutaneus fistula secondary to surgery for lysis of adhesions. After closing the small bowel fistula, the small area of the bowel was wrapped with amnion, and notably, patients were discharged on a regular diet only 12 days after surgery. Importantly, Dino et al. (1965) showed that AM from routine deliveries could be sterilized and kept for
6 weeks at $4^{\circ} \mathrm{C}$ and safely used on acute second degree burns and on skin donor sites (Dino et al., 1965). This was one of the first reports which suggested handling procedures for the AM, which in turn fueled even more interest among clinicians in using the AM for treating skin lesions.

In 1972, Trelford et al. (1972) used human amnion in sheep, and confirmed previous findings that as a surface graft, amnion was able to reduce pain, decrease infection, and reduce the loss of fluids. Also in 1972, Robson and his colleagues began to study the use of AM for severe burns and skin injuries. Similar to Trelford, they reported notable pain relief and immediate adherence of the AM dressing to the wound. Interestingly, the membranes were easily peeled off 1 week after application and, according to Robson, spontaneous re-epithelialization had occurred underneath the membranes. Later that decade, in 1977, Trelford-Sauder et al. (1977) reported the successful use of amnion to replace pelvic peritoneum in patients who had exenterative procedures. Following these and other observations, there was an exponential increase of published clinical studies, which reconfirmed the successful application of fetal membranes in diverse clinical indications, including burns (Gruss and Jirsch, 1978; Bose, 1979), ulcers (Troensagaard-Hansen, 1950; Bennett et al., 1980; Subrahmanyam, 1995), surgical reconstruction of the vagina (Dhall, 1984; Nisolle and Donnez, 1992; Georgy and Aziz, 1996), abdominal surgery (Trelford-Sauder et al., 1978; Silverton et al., 1979; Gharib et al., 1996), and other surgical procedures for the prevention of adhesions (Trelford-Sauder et al., 1978; Muralidharan et al., 1991; Young et al., 1991; Arora et al., 1994; Rennekampff et al., 1994). 
The 1990s can be considered the beginning of modern history on the use of AM in ophthalmology (Dua et al., 2004). In this decade, Dr. Tseng, an ophthalmologist from Miami, applied for Human Cell Tissue Products (HCT/P) regulatory status for the use of amniotic tissues in ocular repair. In 1999, Dr. Tseng's proposal was rejected by the US Food and Drug Administration's (FDA) tissue reference group stating "Amniotic membrane for ocular surface reconstruction is considered a tissue under the current code of federal regulations (CFR) at 21 CFR Part 1270, but the intended use would be non-homologous when $21 \mathrm{CFR}$ Part 1271 becomes effective" (Lee and Tseng, 1997; Tseng et al., 1997). Dr. Tseng appealed the ruling and 1 year later, the FDA reversed the prior ruling stating that "Allogeneic amniotic membrane for ocular repair is considered to be a section $361 \mathrm{HCT} / \mathrm{P}$ if the product's advertising is restricted to homologous use for wound covering." The two main points made by the FDA were that it had to be used as a covering and must be acellular, whereas "Amniotic membrane cultured with stem cells for ocular repair is considered to be a biologic product subject to Investigational New Drug and Biologic License Application.” From that point on, the use of AM in ophthalmic surgery catapulted. Nowadays, there are over 45,000 applications used by the ophthalmologists, including conjunctival reconstruction, burn treatment, glaucoma surgery, and wound healing (Fetterolf and Snyder, 2012).

\section{CURRENT CLINICAL APPLICATIONS OF PLACENTA}

The twenty-first century marks another turning point in which the use of cells isolated from different placental regions are being progressively more investigated and used for their therapeutic potential. These studies have paved the way for what are now considered established clinical uses and investigative clinical trials. Placental tissues, and cells derived thereof, are nowadays interesting therapeutic biomaterials currently used in the clinic. The renewed interest in using the AM in a number of applications has been favored by the development of improved techniques for both membrane cryopreservation (Lee and Tseng, 1997) and advanced isolation and culture procedures for cells derived thereof.

\section{Established Clinical Applications}

Recent advances in tissue preservation techniques, accompanied by evidence of therapeutic effects, have resulted in commercially available AM products for use in patients. A repertoire of data shows that these products promote rapid and complete healing of wounds.

Without a doubt, the AM is widely utilized in the field of ophthalmology. The AM is routinely used as a graft, spread onto the ocular surface to treat epithelial defects or ulcers, or as a bandage to cover the ocular surface to promote healing. An infinite number of ocular pathologies are being treated with the AM, such as corneal epithelial defects, corneal ulcers, glaucoma, pterygium, and bullous keratopathy (Dua et al., 2004; Liu et al., 2010; Riau et al., 2010; Kesting et al., 2014). Furthermore, the AM is also making its way in dermatology, where it can be used to cover burns and to treat chronic ulcers (Lo and Pope, 2009), and in multiple surgical procedures, some of which already mentioned, and others, such as the prevention of post-operative adhesions. The increasing number of case studies will surely support the establishment of a routine use also in the latter two applications.

\section{Clinical Trials}

At the time this review was written, 95 clinical trials evaluating placental cells or AM (Figure 2; Tables 1-5) were registered on the NIH Clinical Trials website (https://clinicaltrials.gov).

Surely, there has been much progress since the first documented use of fetal membranes in the early 1900s, and there have been significant advances in the preparation of amniotic and chorionic membranes for clinical use. In fact, there are many companies which commercialize fetal membranes and/or their derivatives, such as Bio-Tissue ${ }^{\circledR}$, a subsidiary of TissueTech ${ }^{\mathrm{TM}}$ (Prokera $^{\circledR}$, Amniograft $^{\circledR}, \quad$ Amnioguard ${ }^{\circledR}$ ), IOP Ophthalmics $\left(\right.$ AmbioDisk $\left.^{\mathrm{TM}}, \mathrm{Ambio}^{\mathrm{TM}}\right)$, MiMedx $^{\circledR}\left(\right.$ Epifix $^{\circledR}$, Amniofix $\left.^{\circledR}\right)$, Liventa Bioscience (AmnioClear ${ }^{\mathrm{TM}}$ ), Amniox Medical (NEOX ${ }^{\circledR}$ CORD $1 \mathrm{~K}$, Clarix $\left.{ }^{\mathrm{TM}} 1 \mathrm{~K}\right)$, and $\mathrm{NuCel}\left(\mathrm{ReNu}^{\mathrm{TM}}\right.$ amniotic allograft), just to name a few.

Recently published reports have provided consistent evidence of the therapeutic benefit of the AM graft. For example, the AM has been successfully used in patients with bullous keratopathy (NCT01926535, completed, Table 1), and the results suggested that the technique is safe and represents an efficient alternative to the use of therapeutic contact lenses for the relief of ocular pain in these patients (Venegas et al., 2014). In a different study (NCT00659308, completed, Table 1), the AM was shown to be comparable to anterior stromal puncture in the management of pain in patients with bullous keratopathy (Paris Fdos et al., 2013). When tested in patients with scleral thinning, the AM was not as effective as lamellar corneal and scleral transplantation (de Farias et al., 2014), but a phase II/III trial is now enrolling patients for further testing (NCT00801073, Table 1).

The use of a dehydrated human amnion/chorion membrane Epifix $^{\circledR}\left(\right.$ MiMedx $\left.^{\circledR}\right)$ has been reported to enhance healing and promote complete epithelialization of diabetic foot ulcers (NCT01552499, completed, Table 2) (Zelen et al., 2014) and to reduce wound size and improve healing also in patients with venous leg ulcers, both when compared to standard treatment (NCT01552447, completed, Table 2) (Serena et al., 2014). This same group also investigated the use of Amniofix ${ }^{\circledR}\left(\mathrm{MiMedx}^{\circledR}\right)$, a dehydrated human amniotic/chorionic membrane refined by a micronization process, in patients with plantar fasciitis (NCT01659827, completed, Table 3) (Zelen et al., 2013). They reported reduced pain and improved physical and mental scores (evaluated by a health survey) versus controls. Interestingly, bovine AM (NCT01895374, completed, Table 2) has been tested for the treatment of laser-induced skin wounds, showing once again quicker epithelialization when compared to hydrocolloidtreated wounds (Min et al., 2014), suggesting that xenogenic material can also be used in wound treatment.

As mentioned above, the interest in using cells isolated from different placental regions intensified in the twenty-first century. Different types of placenta-derived cells and factors are being 


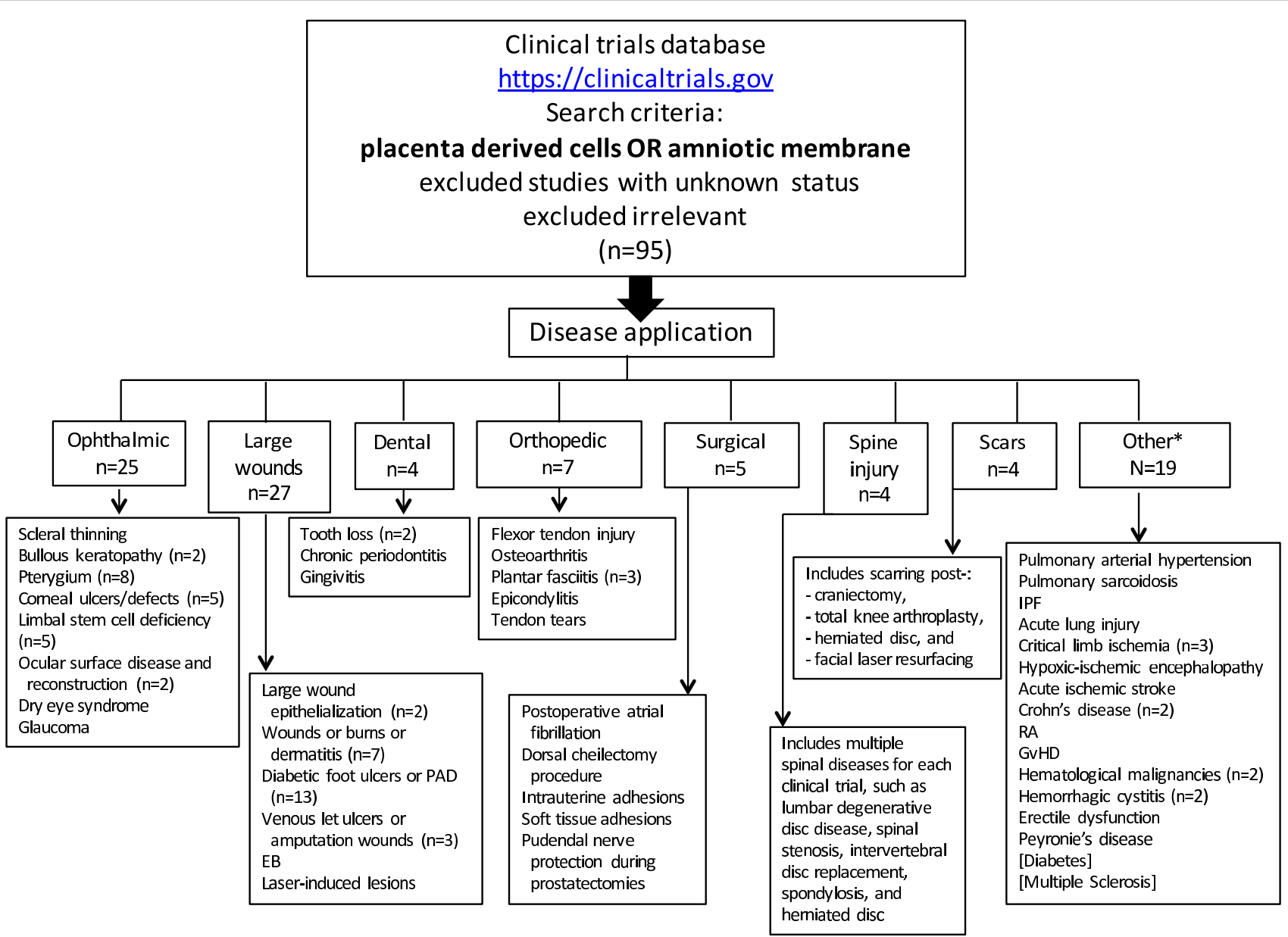

FIGURE 2 | Registered clinical trials using placenta-derived cells or amniotic membrane. The search was performed in June 2015 on the U.S. National Institutes of Health clinical trials website using: placental-derived cells OR amniotic membrane, which resulted in 240 clinical trials. After excluding those with unknown status (which resulted in 196) and irrelevant ones, the search resulted in 95 clinical trials. For each disease application specified in small boxes, $n=1$ unless otherwise specified. PAD, peripheral arterial disease; EB, epidermolysis bullosa; IPF, idiopathic pulmonary fibrosis; RA, rheumatoid arthritis; GvHD, graft versus host disease *Includes two published clinical trials not registered on NIH website (diabetes and multiple sclerosis).

tested in clinical trials. They come from either fetal placental tissues (such as amnion and chorion) or from maternal tissues (such as decidua). For example, PLX-PAD (PLacental eXpanded adherent stromal cells produced by Pluristem Inc.) are isolated from human placenta by enzymatic digestion of both maternal and fetal tissues (Ramot et al., 2009; Kranz et al., 2010). PLX-PAD cells have been used in two clinical trials to treat critical limb ischemia (NCT00919958, completed; NCT00951210, completed), and are now being tested for the treatment of intermittent claudication (NCT01679990, recruiting) and pulmonary arterial hypertension (NCT01795950, active) (Table 5). Thus far, no results have been published for the completed trials.

PDA-001/PDA-002 are culture-expanded mesenchymal-like cells produced by Celgene Cellular Therapeutics. The safety and efficacy of PDA001 cells have been studied in patients with Crohn's Disease (Mayer et al., 2013) (NCT01155362, completed; NCT01769755, completed), ischemic stroke (NCT01310114, terminated by sponsor), pulmonary sarcoidosis (NCT01440192, terminated by sponsor), and active rheumatoid arthritis (NCT01261403, terminated) (Table 5). Published results obtained from the Phase I trial (NCT01769755) in patients with Crohn's disease (Mayer et al., 2013) show that out of the six patients treated with low dose and six with high dose, all low dose and two high dose patients responded to the treatment (defined as $\geq 70$ point decrease from baseline in disease activity score). Some minor evidence of infusion-related events, fever, and headache were reported, but no patient withdrew from the study, and PDA001 was well-tolerated (Mayer et al., 2013). A Phase II study (NCT01155362) investigating PDA001 in treatment-resistant Crohn's disease has just been completed, but results are not yet available. PDA001 cells have also been recently tested in patients with multiple sclerosis (Lublin et al., 2014). The published results showed that in all 16 patients, none developed 5 or more newlesions in 2 consecutive monthly MRI scans during a 6-month follow-up. Some infusion-related adverse events, such as hematoma and swelling, occurred and settled without medication. This study is 
TABLE 1 | Clinical trials using placenta-derived cells, fetal membranes, or derivatives in ophthalmic disorders.

\begin{tabular}{|c|c|c|c|c|c|c|}
\hline Condition & Clinical trial ID & Phase & Materials used & Status & Sponsor & Results/status or remarks \\
\hline Scleral thinning & NCT00801073 & $\|/\| 1 \|$ & AM graft & Enrolling & Federal University of São Paulo, Brazil & $\begin{array}{l}\text { Primary Completion Date: February 2005, last verified December } \\
2008 \text { (de Farias et al., 2014) }\end{array}$ \\
\hline \multirow{2}{*}{$\begin{array}{l}\text { Bullous } \\
\text { keratopathy }\end{array}$} & NCT01926535 & $\|$ & AM graft & Completed & Universidad de Valparaiso, Chile & None available, Study Completion Date: June 2012 \\
\hline & NCT00659308 & ns & AM & Completed & Federal University of São Paulo, Brazil & $\begin{array}{l}\text { Study Completion Date: June 2007, last verified January } 2008 \text { (Paris } \\
\text { Fdos et al., 2013) }\end{array}$ \\
\hline \multirow{8}{*}{$\begin{array}{l}\text { Pterygium or } \\
\text { ocular surface } \\
\text { cicatrizing } \\
\text { diseases or } \\
\text { keratitis }\end{array}$} & NCT02102776 & ns & $\mathrm{AM}$ & Not yet recruiting & Shiyou Zhou, Sun Yat-sen University, China & Estimated Study Completion Date: September 2016 \\
\hline & NCT01319721 & ns & AM graft & Completed & Shiyou Zhou, Sun Yat-sen University, China & $\begin{array}{l}\text { Results available online (https://clinicaltrials.gov), Study Completion } \\
\text { Date: June } 2014\end{array}$ \\
\hline & NCT00457223 & $\|/\| 1 \|$ & AM & Completed & Chulalongkorn University, Thailand & None available, study completed in 2007 \\
\hline & NCT00802620 & 1 & AM & Enrolling & Federal University of São Paulo, Brazil & $\begin{array}{l}\text { None available, Primary Completion Date: June 2005, last verified } \\
\text { December } 2008\end{array}$ \\
\hline & NCT02015000 & ns & AM & Recruiting & National Taiwan University Hospital, Taiwan & $\begin{array}{l}\text { Estimated Study Completion Date: November 2014, last verified } \\
\text { December } 2013\end{array}$ \\
\hline & NCT00383825 & ns & AM & Completed & Baskent University, Ankara, Turkey & $\begin{array}{l}\text { None available, Estimated Study Completion Date: December 2004, } \\
\text { last verified October } 2006\end{array}$ \\
\hline & NCT00344201 & I & AM & Completed & Singapore National Eye Centre, Singapore & None available, Study Completion Date: January 2008 \\
\hline & NCT02116062 & ns & AM & Recruiting & University Hospital, Strasbourg, France & Estimated Study Completion Date: March 2016 \\
\hline \multirow{5}{*}{$\begin{array}{l}\text { Corneal wounds } \\
\text { or ulcers or } \\
\text { epithelial defects }\end{array}$} & NCT00915759 & ns & ProKera $^{\circledR}$ & Active & $\begin{array}{l}\text { Walter Reed National Military Medical Center, } \\
\text { Washington, DC, USA }\end{array}$ & $\begin{array}{l}\text { Estimated Study Completion Date: December 2014, last verified } \\
\text { July } 2014\end{array}$ \\
\hline & NCT02168790 & 0 & $\begin{array}{l}\text { Amnioclip ring } \\
\text { system }\end{array}$ & Completed & Klinikum Chemnitz gGmbH, Germany & None available, Study Completion Date: August 2013 \\
\hline & NCT00238862 & ns & AM & Completed & $\begin{array}{l}\text { King Khaled Eye Specialist Hospital, Riyadh, } \\
\text { Saudi Arabia }\end{array}$ & None available, study completed in 2007 \\
\hline & NCT02395952 & ns & $\begin{array}{l}\text { ProKera } \\
\text { Ambiodisk }\end{array}$ & Not yet recruiting & Milton S. Hershey Medical Center, PA, USA & Estimated Primary Completion Date: February 2018 \\
\hline & NCT02148016 & $1 / 11$ & AM & Recruiting & Sun Yat-sen University, China & $\begin{array}{l}\text { Estimated Study Completion Date: September 2014, last verified } \\
\text { May } 2014\end{array}$ \\
\hline \multirow[t]{5}{*}{$\begin{array}{l}\text { Limbal stem cell } \\
\text { deficiency }\end{array}$} & NCT01377311 & I & AM & Terminated & National Taiwan University Hospital, Taiwan & $\begin{array}{l}\text { Study terminated since technique not used in patients, last verified } \\
\text { June } 2011\end{array}$ \\
\hline & NCT00736307 & I/II & AM & Completed & Royan Institute, Tehran, Iran & $\begin{array}{l}\text { None available, Study Completion Date: October 2009, last verified } \\
\text { April } 2010\end{array}$ \\
\hline & NCT01562002 & $1 / 11$ & AM & Completed & $\begin{array}{l}\text { Instituto Universitario de Oftalmobiología } \\
\text { Aplicada, Spain }\end{array}$ & $\begin{array}{l}\text { None available, Study Completion Date: December 2014, last verified } \\
\text { January } 2015\end{array}$ \\
\hline & NCT01619189 & $\|$ & AM & Ongoing & $\begin{array}{l}\text { Centre Hospitalier National d'Ophtalmologie } \\
\text { des Quinze-Vingts, France }\end{array}$ & $\begin{array}{l}\text { Primary Completion Date: December 2014, last verified } \\
\text { February } 2015\end{array}$ \\
\hline & NCT00491959 & । & AM & Terminated & National Taiwan University Hospital, Taiwan & $\begin{array}{l}\text { Study terminated due to unstable cell sheet quality, thus this } \\
\text { technique was not used on patients }\end{array}$ \\
\hline \multirow{2}{*}{$\begin{array}{l}\text { Ocular surface } \\
\text { disease and } \\
\text { reconstruction }\end{array}$} & NCT00348114 & $\|$ & AM & Completed & Singapore National Eye Centre, Singapore & This study has suspended participant recruitment since 2006 \\
\hline & NCT01341223 & ns & AM & Recruiting & National Taiwan University Hospital, Taiwan & $\begin{array}{l}\text { Estimated Primary Completion Date: March 2016, last verified } \\
\text { March } 2012\end{array}$ \\
\hline $\begin{array}{l}\text { Dry eye } \\
\text { syndrome }\end{array}$ & NCT02369861 & I & ACCS & Recruiting & Stemnion, Inc., Pittsburgh, PA, USA & Estimated Study Completion Date: December 2015 \\
\hline Glaucoma & NCT01551550 & $\|/\| 1 \|$ & AM graft & Recruiting & Tissue Tech'T Inc., Miami, FL, USA & Estimated Study Completion Date: August 2015 \\
\hline
\end{tabular}

The search status as of June 2015 registered on the ClinicalTrials. gov site of U.S. National Institutes of Health (https://Clinicaltrials. gov).

ns, not specified; AM, amniotic membrane; ACCS, amnion-derived Cellular Cytokine Solution (Stemnion, Inc.); ProKera ${ }^{\circledast}$ (Tissue Tech ${ }^{\mathrm{TM}}$ Inc.) is a device made by a piece of amniotic membrane tissue in between two rings made out of flexible material. 


\begin{tabular}{|c|c|c|c|c|c|c|}
\hline Condition & Clinical trial ID & Phase & Materials used & Status & Sponsor & Results/status or remarks \\
\hline \multirow{2}{*}{$\begin{array}{l}\text { Large wound } \\
\text { epithelialization }\end{array}$} & NCT01824381 & । & AM & Recruiting & Red de Terapia Celular, Spain & Estimated Study Completion Date: July 2015, last verified April 2013 \\
\hline & NCT01948934 & I & AM & Recruiting & $\begin{array}{l}\text { Fundacion para la Formacion e Investigacion } \\
\text { Sanitarias de la Region de Murcia, Spain }\end{array}$ & Estimated Study Completion Date: June 2015, last verified March 2014 \\
\hline \multirow{7}{*}{$\begin{array}{l}\text { Wounds or } \\
\text { burns or } \\
\text { dermatitis }\end{array}$} & NCT00674999 & $\|/\|$ & Amnion & Withdrawn & $\begin{array}{l}\text { The University of Texas Medical Branch, } \\
\text { Galveston, TX, USA }\end{array}$ & $\begin{array}{l}\text { Study was withdrawn prior to enrolling due to skin bank being destroyed by } \\
\text { hurricane }\end{array}$ \\
\hline & NCT00592189 & 0 & Amnion & Completed & $\begin{array}{l}\text { The University of Texas Medical Branch, } \\
\text { Galveston, TX, USA }\end{array}$ & None available, Study Completion Date: June 2014, last verified June 2014 \\
\hline & NCT00886470 & $1 / 11$ & ACCS & Terminated & Stemnion, Inc., Pittsburgh, PA, USA & This study was terminated early due to slow accrual of patients \\
\hline & NCT01715012 & ॥ & ACCS & Terminated & Stemnion, Inc., Pittsburgh, PA, USA & This study was terminated early due to enrollment futility \\
\hline & NCT02389777 & $\|$ & ACCS & Not yet recruiting & Stemnion, Inc., Pittsburgh, PA, USA & Estimated Study Completion Date: March 2016 \\
\hline & NCT01714973 & I & ACCS & Active & Stemnion, Inc., Pittsburgh, PA, USA & Estimated Study Completion Date: October 2015 \\
\hline & NCT02314416* & IV & $\begin{array}{l}\text { Amniotic stem } \\
\text { cells }\end{array}$ & Terminated & $\begin{array}{l}\text { Georgia Regents University, Augusta, GA, } \\
\text { USA }\end{array}$ & This study has been withdrawn prior to enrollment \\
\hline \multirow{13}{*}{$\begin{array}{l}\text { Diabetic foot } \\
\text { ulcers or } \\
\text { peripheral } \\
\text { arterial disease } \\
\text { or diabetes }\end{array}$} & NCT02344329 & IV & Amnion & Recruiting & University of North Dakota, USA & Estimated Study Completion Date: August 2016, last verified January 2015 \\
\hline & NCT01859117 & I & PDA002 & Active & Celgene Corporation, NJ, USA & Ongoing, not recruiting, estimated completion data June 2016 \\
\hline & NCT02460081 & ॥ & PDA002 & Recruiting & Celgene Corporation, NJ, USA & Estimated Study Completion Date: September 2017 \\
\hline & NCT02264288 & ॥ & PDA002 & Recruiting & Celgene Corporation, NJ, USA & Estimated Study Completion Date: June 2018 \\
\hline & NCT02399826 & ns & AM & Recruiting & $\begin{array}{l}\text { Lower Extremity Institute for Research and } \\
\text { Therapy, OH, USA }\end{array}$ & Estimated Study Completion Date: January 2016 \\
\hline & NCT02461641 & ns & NuShield, Affinity & Recruiting & NuCel, LLC, Birmingham, AL, USA & Estimated Study Completion Date: September 2016 \\
\hline & NCT02209051 & IV & AMNIOEXCEL & Ongoing & Derma Sciences, Inc., NJ, USA & Estimated Study Completion Date: August 2015 \\
\hline & NCT01693133 & ns & EpiFix $^{\oplus}$ & Recruiting & MiMedx ${ }^{\oplus}$ Group, Inc., Marietta, GA, USA & Estimated Study Completion Date: July 2015, last verified March 2015 \\
\hline & NCT01552499 & ns & EpiFix $^{\circledast}$ & Completed & MiMedx Group, Inc., Marietta, GA, USA & $\begin{array}{l}\text { Study Completion Date: August 2012, last verified September } 2012 \\
\text { (Zelen et al., 2014) }\end{array}$ \\
\hline & NCT01657474 & ns & EpiFix ${ }^{\oplus}$ & Completed & MiMedx ${ }^{\circledast}$ Group, Inc., Marietta, GA, USA & $\begin{array}{l}\text { None available, Study Completion Date: November 2013, last verified } \\
\text { December } 2013\end{array}$ \\
\hline & NCT01921491 & ns & EpiFix $^{\oplus}$ & Recruiting & MiMedx Group, Inc., Marietta, GA, USA & Estimated Primary Completion Date: May 2015 \\
\hline & NCT02120755 & IV & AmnioClearTM & Not yet recruiting & Liventa Bioscience, Pennsylvania, USA & Estimated Primary Completion Date: January 2015, last verified April 2014 \\
\hline & NCT02166294 & ns & NEOX ${ }^{\circledR}$ CORD $1 \mathrm{~K}$ & Recruiting & Amniox Medical, Inc., Atlanta, GA, USA & Estimated Study Completion Date: June 2015, last verified July 2014 \\
\hline \multirow{3}{*}{$\begin{array}{l}\text { Venous leg } \\
\text { ulcers or } \\
\text { amputation } \\
\text { wounds }\end{array}$} & NCT01552447 & ns & Epifix ${ }^{\oplus}$ & Completed & MiMedx ${ }^{\circledast}$ Group, Inc., Marietta, GA, USA & $\begin{array}{l}\text { Study Completion Date: May 2014, last verified November } 2013 \\
\text { (Serena et al., 2014) }\end{array}$ \\
\hline & NCT02011503 & ns & Epifix ${ }^{\circledast}$ & Recruiting & MiMedx ${ }^{\otimes}$ Group, Inc., Marietta, GA, USA & Estimated Study Completion Date: January 2016 \\
\hline & NCT00820274 & $\|$ & AM & Terminated & University Hospital, Limoges, France & Clinical trial encountered difficulties enrolling a sufficient number of patients \\
\hline $\begin{array}{l}\text { Epidermolysis } \\
\text { bullosa }\end{array}$ & NCT02286427 & III & AM & Recruiting & $\begin{array}{l}\text { Assistance Publique - Hôpitaux de Paris, } \\
\text { France }\end{array}$ & Estimated Study Completion Date: January 2019 \\
\hline $\begin{array}{l}\text { Laser- } \\
\text { treatment } \\
\text { induced } \\
\text { lesions }\end{array}$ & NCT01895374 & ns & Bovine AM & Completed & $\begin{array}{l}\text { Seoul National University Hospital, } \\
\text { South Korea }\end{array}$ & Study Completion Date: May 2013, last verified July 2013 (Min et al., 2014) \\
\hline
\end{tabular}

The search status as of June 2015 registered on the ClinicalTrials.gov site of U.S. National Institutes of Health (https://clinicaltrials. gov).

ns, not specified; AM, amniotic membrane; ACCS, amnion-derived Cellular Cytokine Solution (Stemnion, Inc.); NuShield (NuCel, LLC) is a sterilized dehydrated amnion chorion membrane patch, affinity is an aseptically produced,

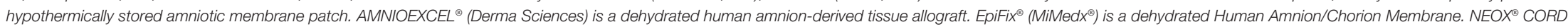
(Amniox Medical) is made from cryopreserved human amniotic membrane and umbilical cord. AmnioClearTM (Liventa Bioscience) is an allograft membrane comprising of the amnion and chorion. 
TABLE 3 | Clinical trials using placenta-derived cells, fetal membranes, or derivatives in dental and orthopedic defects.

\begin{tabular}{|c|c|c|c|c|c|c|c|}
\hline Condition & & Clinical trial ID & Phase & Materials used & Status & Sponsor & Results/status or remarks \\
\hline \multirow[t]{4}{*}{ Dental defects } & Tooth loss & NCT01836783 & ns & Amnion & Recruiting & $\begin{array}{l}\text { University of Alabama at Birmingham, } \\
\text { USA }\end{array}$ & Estimated Study Completion Date: April 2016 \\
\hline & & NCT02482987 & ns & BioXclude ${ }^{\mathrm{TM}}$ & $\begin{array}{l}\text { Not yet } \\
\text { recruiting }\end{array}$ & $\begin{array}{l}\text { Eisenhower Army Medical Center, } \\
\text { GA, USA }\end{array}$ & Estimated Study Completion Date: July 2018 \\
\hline & $\begin{array}{l}\text { Chronic } \\
\text { periodontitis }\end{array}$ & NCT02033226 & III & AM & Completed & $\begin{array}{l}\text { SVS Institute of Dental Sciences, } \\
\text { India }\end{array}$ & $\begin{array}{l}\text { None available, Study Completion Date: September 2013, last } \\
\text { verified January } 2014\end{array}$ \\
\hline & Gingivitis & NCT02071199 & I & ACCS & Recruiting & Stemnion, Inc., Pittsburgh, PA, USA & Estimated Study Completion Date: August 2015 \\
\hline \multirow[t]{7}{*}{$\begin{array}{l}\text { Orthopedic } \\
\text { defects }\end{array}$} & $\begin{array}{l}\text { Flexor tendon } \\
\text { injury }\end{array}$ & NCT02361814 & ns & AM graft & Recruiting & University of Tampere, Finland & Estimated Study Completion Date: December 2016 \\
\hline & Osteoarthritis & NCT02318511 & ns & $\begin{array}{l}\text { ReNu amniotic } \\
\text { allograft }\end{array}$ & Recruiting & NuCel, LLC, Birmingham, AL, USA & Estimated Study Completion Date: March 2017 \\
\hline & Plantar fasciitis & NCT02427191 & $\|/\|$ & AmnioFix ${ }^{\circledR}$ & Recruiting & $\begin{array}{l}\text { MiMedx } x^{\circledast} \text { Group, Inc., Marietta, GA, } \\
\text { USA }\end{array}$ & Estimated Study Completion Date: December 2017 \\
\hline & & NCT01659827 & ns & AmnioFix ${ }^{\circledR}$ & Completed & $\begin{array}{l}\text { MiMedx Group, Inc., Marietta, GA, } \\
\text { USA }\end{array}$ & $\begin{array}{l}\text { Study Completion Date: March 2013, last verified December } 2013 \\
\text { (Zelen et al., 2013) }\end{array}$ \\
\hline & & NCT01996111 & ns & EpiFix ${ }^{\circledast}$ & Terminated & $\begin{array}{l}\text { MiMedx } x^{\circledast} \text { Group, Inc., Marietta, GA, } \\
\text { USA }\end{array}$ & Study terminated due to change in regulatory status \\
\hline & Epicondylitis & NCT01921569 & ns & $\begin{array}{l}\text { Micronized } \\
\text { dHACM } \\
\text { suspension }\end{array}$ & Terminated & $\begin{array}{l}\text { MiMedx } x^{\circledast} \text { Group, Inc., Marietta, GA, } \\
\text { USA }\end{array}$ & $\begin{array}{l}\text { Study terminated by sponsor, used micronized dehydrated human } \\
\text { amniotic membrane (dHACM) suspension }\end{array}$ \\
\hline & Tendon tears & NCT01708187 & ns & Clarix ${ }^{\mathrm{TM}} 1 \mathrm{k}$ & Terminated & $\begin{array}{l}\text { Orthopedic Foot and Ankle Center, } \\
\text { OH, USA }\end{array}$ & Protocol halted due to less than anticipated recruitment \\
\hline
\end{tabular}

The search status as of June 2015 registered on the ClinicalTrials. gov site of U.S. National Institutes of Health (https://clinicaltrials. gov).

ns, not specified; AM, amniotic membrane; dHACM, dehydrated human amniotic membrane; PAD, peripheral artery disease; ACCS, amnion-derived Cellular Cytokine Solution (Stemnion, Inc.); BioXClude

CO, USA) is a human amnion chorion allograft. ReNuTM (NuTech Medical) is a bioactive suspension derived from human amnion and amniotic fluid. AmnioFix (MiMedx') is a composite amniotic tissue membrane. PLX-PAD are

PLacental eXpanded adherent stromal cells (produced by Pluristem Inc.), Clarix ${ }^{\mathrm{TM}}$ 1k (Amniox Medical), C-HAM is a cryopreserved Human Amniotic Membrane. 
TABLE 4 | Clinical trials using placenta-derived cells, fetal membranes, or derivatives in surgery, spinal injuries, and scarring.

\begin{tabular}{|c|c|c|c|c|c|c|c|}
\hline Condition & & Clinical trial ID & Phase & Materials used & Status & Sponsor & Results/status or remarks \\
\hline \multirow[t]{5}{*}{ Surgical } & Post-operative atrial fibrillation & NCT02193321 & $1 / 11$ & AM patch & Recruiting & University of Arizona, USA & $\begin{array}{l}\text { Estimated Study Completion Date: July 2015, } \\
\text { last verified July } 2014\end{array}$ \\
\hline & $\begin{array}{l}\text { Dorsal cheilectomy procedure for Hallux } \\
\text { Rigidus }\end{array}$ & NCT01825356 & IV & AM & Recruiting & $\begin{array}{l}\text { OrthoCarolina Research Institute, Inc., } \\
\text { Charlotte, NC, USA }\end{array}$ & $\begin{array}{l}\text { Estimated Study Completion Date: } \\
\text { December 2016, last verified December } 2014\end{array}$ \\
\hline & Intrauterine adhesions & NCT02132104 & ns & AM graft & $\begin{array}{l}\text { Not yet } \\
\text { recruiting }\end{array}$ & Capital Medical University, Beijing, China & $\begin{array}{l}\text { Estimated Study Completion Date: } \\
\text { November } 2016\end{array}$ \\
\hline & $\begin{array}{l}\text { Adhesions of soft tissue during the } \\
\text { removal of segmental posterior lumbar } \\
\text { instrumentation }\end{array}$ & NCT01357187 & ns & AmnioFix ${ }^{\circledast}$ & Completed & MiMedx Group, Inc., Marietta, GA, USA & $\begin{array}{l}\text { none available, Study Completion Date: } \\
\text { May 2014, last verified June } 2014\end{array}$ \\
\hline & $\begin{array}{l}\text { Pudendal nerve protection during } \\
\text { laparoscopic prostatectomies }\end{array}$ & NCT01832168 & ns & AmnioFix ${ }^{\otimes}$ & Completed & MiMedx Group, Inc., Marietta, GA, USA & $\begin{array}{l}\text { None available, Study Completion Date: } \\
\text { June 2014, last verified March } 2015\end{array}$ \\
\hline \multirow[t]{4}{*}{$\begin{array}{l}\text { Spinal } \\
\text { injuries }\end{array}$} & Various spinal injuries ${ }^{a}$ & NCT02070484 & ns & $\mathrm{NuCel}^{\circledast}$ & Recruiting & OhioHealth, Colombus, OH,USA & $\begin{array}{l}\text { Estimated Study Completion Date: February } \\
\text { 2016, last verified April } 2014\end{array}$ \\
\hline & & NCT02381067 & ns & $\mathrm{NuCel}^{\circledast}$ & Recruiting & NuCel, LLC, Birmingham, AL, USA & $\begin{array}{l}\text { Estimated Study Completion Date: September } \\
2016\end{array}$ \\
\hline & & NCT02023372 & ns & NuCel $\left.\right|^{\otimes}$ & Recruiting & NuCel, LLC, Birmingham, AL, USA & Estimated Study Completion Date: July 2017 \\
\hline & Spinal stenosis and herniated disk & NCT02380456 & ns & EpiFix $^{\circledR}$ & Recruiting & $\begin{array}{l}\text { Alexander P. Hughes, MD and MiMedx } \\
\text { Group, Inc., Marietta, GA, USA }\end{array}$ & $\begin{array}{l}\text { Estimated Study Completion Date: December } \\
2017\end{array}$ \\
\hline \multirow[t]{4}{*}{ Scarring } & Scarring post craniectomy & NCT02033824 & ns & EpiFix ${ }^{\oplus}$ & Recruiting & MiMedx Group, Inc., Marietta, GA, USA & Estimated Study Completion Date: June 2016 \\
\hline & Scarring post total knee arthroplasty & NCT02088567 & ns & EpiFix ${ }^{\oplus}$ & Completed & MiMedx Group, Inc., Marietta, GA, USA & $\begin{array}{l}\text { None available, Study Completion Date: } \\
\text { October 2014, last verified March } 2015\end{array}$ \\
\hline & Scarring/spinal stenosis/herniated disk & NCT02300909 & IV & $\mathrm{EpiFix}^{\oplus}$ & Recruiting & MiMedx Group, Inc., Marietta, GA, USA & $\begin{array}{l}\text { Estimated Study Completion Date: December } \\
2017\end{array}$ \\
\hline & Scarring post laser resurfacing of the face & NCT01995604 & ns & $\mathrm{EpiFix}^{\circledR}$ & Terminated & MiMedx Group, Inc., Marietta, GA, USA & This study was withdrawn prior to enrollment \\
\hline
\end{tabular}

The search status as of June 2015 registered on the ClinicalTrials. gov site of U.S. National Institutes of Health (https://Clinicaltrials. gov).

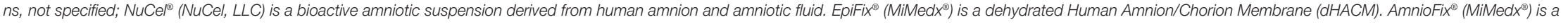
composite amniotic tissue membrane.

alncludes: lumbar degenerative disk disease, spinal stenosis, spondylolisthesis, spondylosis, invertebral disk displacement and degeneration, spinal diseases, bone disease. 
TABLE 5 | Clinical trials using placenta-derived cells, fetal membranes, or derivatives in other disorders.

\begin{tabular}{|c|c|c|c|c|c|c|c|}
\hline Condition & & Clinical trial ID & Phase & Materials used & Status & Sponsor & Results/status or remarks \\
\hline \multirow[t]{4}{*}{ Lung diseases } & Pulmonary arterial hypertension & NCT01795950 & । & PLX-PAD & Recruiting & Pluristem Inc., Haifa, Israel & Estimated Study Completion Date: September 2016 \\
\hline & Pulmonary sarcoidosis & NCT01440192 & I & PDA001 & Terminated & Celgene Corporation, NJ, USA & Study terminated by sponsor \\
\hline & Idiopathic pulmonary fibrosis & NCT01385644 & 1 & Placental MSC & Completed & $\begin{array}{l}\text { The Prince Charles Hospital, } \\
\text { Brisbane, QLD, Australia }\end{array}$ & Study Completion Date: May 2013 (Chambers et al., 2014) \\
\hline & Acute lung injury & NCT02175303 & $1 / 11$ & $\begin{array}{l}\text { Decidual stromal } \\
\text { cells }\end{array}$ & Recruiting & $\begin{array}{l}\text { Karolinska Institutet, Stockholm, } \\
\text { Sweden }\end{array}$ & Estimated Study Completion Date: December 2017 \\
\hline \multirow{5}{*}{$\begin{array}{l}\text { Ischemic } \\
\text { diseases }\end{array}$} & \multirow{3}{*}{$\begin{array}{l}\text { PAD/peripheral vascular disease/ } \\
\text { critical limb ischemia }\end{array}$} & NCT00951210 & । & PLX-PAD & Completed & Pluristem Inc., Haifa, Israel & None available, Study Completion Date: October 2011 \\
\hline & & NCT01679990 & ॥ & PLX-PAD & Recruiting & Pluristem Inc., Haifa, Israel & Estimated Primary Completion Date: December 2015 \\
\hline & & NCT00919958 & । & PLX-PAD & Completed & Pluristem Inc., Haifa, Israel & None available, Study Completion Date: June 2012 \\
\hline & $\begin{array}{l}\text { Severe hypoxic-ischemic } \\
\text { encephalopathy in neonates }\end{array}$ & NCT02434965 & $\|$ & HPDSC & $\begin{array}{l}\text { Not yet } \\
\text { recruiting }\end{array}$ & $\begin{array}{l}\text { New York Medical College, NY, } \\
\text { USA }\end{array}$ & Estimated Study Completion Date: December 2019 \\
\hline & Acute ischemic stroke & NCT01310114 & $\|$ & PDA001 & Terminated & Celgene Corporation, NJ, USA & Study terminated by sponsor \\
\hline \multirow[t]{6}{*}{$\begin{array}{l}\text { Autoimmune } \\
\text { diseases }\end{array}$} & \multirow[t]{2}{*}{ Crohn's disease } & NCT01155362 & $\|$ & PDA001 & Completed & Celgene Corporation, NJ, USA & $\begin{array}{l}\text { None available, Study Completion Date: April 2014, last } \\
\text { verified July } 2014\end{array}$ \\
\hline & & NCT01769755 & I & PDA001 & Completed & Celgene Corporation, NJ, USA & $\begin{array}{l}\text { Study Completion Date: November 2014, last verified April } \\
2015 \text { (Mayer et al., 2013) }\end{array}$ \\
\hline & Rheumatoid arthritis & NCT01261403 & $\|$ & PDA001 & Terminated & Celgene Corporation, NJ, USA & Enrollment terminated pending additional Phase 1 data \\
\hline & Graft versus host disease & NCT02172924 & $1 / 11$ & $\begin{array}{l}\text { Decidual stromal } \\
\text { cells }\end{array}$ & $\begin{array}{l}\text { Not yet } \\
\text { recruiting }\end{array}$ & $\begin{array}{l}\text { Karolinska Institutet, Stockholm, } \\
\text { Sweden }\end{array}$ & Estimated Study Completion Date: December 2019 \\
\hline & Multiple sclerosis & Not applicable & - & PDA001 & $\begin{array}{l}\text { Published } \\
\text { study }\end{array}$ &  & Lublin et al. (2014) \\
\hline & Diabetes & Not applicable & - & $\begin{array}{l}\text { Placenta-derived } \\
\text { MSC }\end{array}$ & $\begin{array}{l}\text { Published } \\
\text { study }\end{array}$ & - & Jiang et al. (2011) \\
\hline \multirow[t]{2}{*}{$\begin{array}{l}\text { Hematological } \\
\text { malignancies }\end{array}$} & Various $^{\mathrm{a}}$ & NCT00596999 & 1 & HPDSC & Enrolling & Celgene Corporation, NJ, USA & $\begin{array}{l}\text { Estimated Study Completion Date: December 2013, last } \\
\text { verified November } 2007\end{array}$ \\
\hline & Various $^{b}$ & NCT01586455 & 1 & HPDSC & Recruiting & $\begin{array}{l}\text { New York Medical College, } \\
\text { NY, USA }\end{array}$ & Estimated Study Completion Date: December 2019 \\
\hline \multirow[t]{2}{*}{$\begin{array}{l}\text { Hemorrhagic } \\
\text { cystitis }\end{array}$} & - & NCT02172963 & $1 / \|$ & $\begin{array}{l}\text { Decidual stromal } \\
\text { cells }\end{array}$ & Completed & $\begin{array}{l}\text { Karolinska Institutet, Stockholm, } \\
\text { Sweden }\end{array}$ & None available, study completed December 2013 \\
\hline & - & NCT02174536 & $\|$ & $\begin{array}{l}\text { Decidual stromal } \\
\text { cells }\end{array}$ & Recruiting & $\begin{array}{l}\text { Karolinska Institutet, Stockholm, } \\
\text { Sweden }\end{array}$ & Estimated Study Completion Date: December 2016 \\
\hline $\begin{array}{l}\text { Peyronie's } \\
\text { disease }\end{array}$ & - & NCT02395029 & 1 & PMD-MSC & Completed & $\begin{array}{l}\text { Melissa Marchand from Z Urology, } \\
\text { Coral Springs, FL, USA }\end{array}$ & None available, Study completed in March 2015 \\
\hline $\begin{array}{l}\text { Erectile } \\
\text { dysfunction }\end{array}$ & - & NCT02398370 & । & PMD-MSC & Completed & $\begin{array}{l}\text { Melissa Marchand from Z Urology, } \\
\text { Coral Springs, FL, USA }\end{array}$ & None available, Study completed in March 2015 \\
\hline
\end{tabular}

The search status as of June 2015 registered on the ClinicalTrials.gov site of U.S. National Institutes of Health (https://clinicaltrials.gov).

ns, not specified; AM, amniotic membrane; PMD-MSC, placental matrix-derived mesenchymal stem cells; PLX-PAD are PLacental eXpanded adherent stromal cells (produced by Pluristem Inc.), Clarix'M $1 \mathrm{~K}$ (Amniox Medical), HPDSC (human placenta-derived stem cells produced by Celgene Cellular Therapeutics), PDA001 are human placenta-derived adherent cells (Celgene Corporation).

a/ncludes: myelodysplastic syndrome, acute myelogenous/lymphocytic, leukemia, sickle cell disease, beta thalassemia, inborn errors of metabolism, severe combined immunodeficiency disease.

IIncludes: mucopolysaccharidosis I, mucopolysaccharidosis VI, adrenoleukodystrophy, Niemann-Pick disease, metachromatic leukodystrophy, Wolman disease, Krabbe's disease, Gaucher's disease, fucosidosis, Batten disease, severe aplastic anemia, Diamond-Blackfan anemia, amegakaryocytic thrombocytopenia, myelodysplastic syndrome, acute myelogenous leukemia, acute lymphocytic leukemia. 
important in underlining the safety of placental cells in treating patients with multiple sclerosis, and encourages future studies to investigate therapeutic benefit in this physical and mental disabling condition.

PDA002 cells are currently being tested for the treatment of patients with diabetic foot ulcers and peripheral arterial disease (NCT01859117, active; NCT02460081, recruiting; NCT02264288, recruiting) (Table 2). Human placenta-derived stem cells (HPDSC, also produced by Celgene Cellular Therapeutics) are obtained from placenta perfusion after removal non-viable and red blood cells and tissue debris. These cells are cryopreserved without culture expansion (Kang et al., 2013). HPDSC will soon be investigated for treating neonates with severe hypoxic-ischemic encephalopathy (NCT02434965, not yet recruiting), and patients with hematological malignancies (NCT00596999, enrolling; NCT01586455, recruiting) (Table 5).

Placental-derived MSC are isolated from the placental tissues after the removal of umbilical cord and external membranes by enzymatic digestions (Prince Charles Hospital in Brisbane, Australia) (Brooke et al., 2009). The safety of placental-derived MSC has been studied in patients with idiopathic pulmonary fibrosis (IPF, NCT01385644, completed). The results of this study have recently been published and show that intravenous infusion of 2 million cells per kilogram is safe in patients with moderate to severe IPF. Of note, the possibility of embolization of cells in the compromised pulmonary vascular bed, a major concern during this type of treatment, did not lead to major adverse clinical outcomes. Only minor, transient changes in hemodynamics and gas exchange, and only minor adverse events were observed (Chambers et al., 2014).

Placenta-derived decidual stromal cells can be isolated by either trypsin digestion or tissue explants (Karolinska Institutet, Sweden). These cells derive from the decidua parietalis and are of maternal origin (Ringden et al., 2013; Erkers et al., 2015). They have been tested in a pilot study in patients with hemorrhagic cystitis (NCT02172963, completed) and will soon be investigated in a different clinical trial to treat the same disease (NCT02174536, recruiting) (Table 5). A clinical trial is also due to begin for the treatment of patients with Graft versus Host Disease (NCT02172924, not yet recruiting). Moreover, a pilot study is currently recruiting patients with acute lung injury to evaluate the safety of placenta DSCs (NCT02175303, recruiting) (Table 5).

Remarkably, encouraging results have been published for studies using placenta-derived cells in patients with types II diabetes (Table 5) (Jiang et al., 2011). In this Phase I study aimed at evaluating the safety of placenta-derived MSC, 10 patients with type 2 diabetes were given 3 intravenous infusions at the 1-month intervals. The authors reported that the mean insulin requirement was significantly reduced at the 3-month follow-up, and no side effects (fever, chills, liver damage) were documented. This trial could represent a turning point in the use of placental cells, since it is the first to document their use in patients with diabetes. A different study of particular interest was recently published investigating the immunogenicity of placenta decidual stromal cells in combination with AM in an 11-month-old patient with epidermolysis bullosa (Kaipe et al.,
2015), showing improved healing of blisters and wounds, but at the same time warranting further investigations on the immunogenicity of these cells.

Notably, other clinical trials have and are currently investigating placental cell derivatives and, in particular amnion-derived cellular cytokine solution (ACCS) (Stemnion, Inc., Pittsburgh, PA, USA). ACCS is obtained from amniotic cell culture (Bergmann et al., 2009), and contains factors relevant for wound healing, such as platelet-derived growth factor (PDGF), vascular endothelial growth factor (VEGF), angiogenin, TGF- $\beta 2$, TIMP-1, and TIMP-2 (Steed et al., 2008). It is currently being tested in patients with radiation-induced dermatitis (NCT01714973, active), and a separate trial is due to begin in patients with UV-induced burns (NCT02389777, not yet recruiting), (Table 2). Moreover, other trials are currently recruiting patients to test ACCS in dry eye syndrome (NCT02369861, recruiting, Table 1), and gingivitis (NCT02071199, recruiting, Table 3). Two trials, the first aimed at investigating ACCS in partial thickness wounds (NCT00886470), and the second in patients with deep burns (NCT01715012), were terminated due to difficulties in enrolling patients (Table 2).

Another placenta-derived product, amniotic membrane extract (AMX), which is based on lyophilized human AM for topical application, is under investigation in persistent corneal epithelial defects and has shown promising results for reducing epithelial defects (Kordić et al., 2013).

\section{MECHANISMS OF ACTION: THE QUEST FOR SCIENTIFIC RATIONALE}

Evidence of long-term survival with no signs of immune reaction was provided many years ago when the AM was used as an allograft under skin (Douglas et al., 1954) or in the peritoneal cavity (Trelford et al., 1974). Subsequently, a glycoprotein from amnion was reported to be responsible for suppressing "foreign body" reactions by acting on lymphocytes and preventing lymphoblastogenesis (McIntyre and Faulk, 1979).

Since then, numerous mechanisms have been put forth, either to explain the therapeutic effects of the intact or decellularized AM or those of isolated placental cells. Nowadays, the clinical potential of placenta-derived cells essentially relies on their paracrine mechanisms able to induce anti-inflammatory responses and re-epithelialization, and also to possess pro- or anti-angiogenic properties. Below we will briefly discuss these aspects.

\section{Anti-Inflammatory Properties}

Among the paracrine actions underlying the anti-inflammatory effect of placenta-derived cells are their interactions with immune cells of innate and adaptive immunity. Indeed, many studies have reported the ability of placenta-derived cells to suppress the proliferation of activated T cells (Bailo et al., 2004; Chang et al., 2006; Wolbank et al., 2007; Prasanna et al., 2010; Kronsteiner et al., 2011a,b), reduce Th1 inflammatory cytokines and induce T regulatory cells (Raicevic et al., 2011; Ohshima et al., 2012; Anam et al., 2013; Parolini et al., 2014; Pianta et al., 2015), and target B lymphocytes (Li et al., 2005; Ma et al., 2012). Furthermore, they can 
also influence antigen presenting cells by blocking differentiation of monocytes to dendritic cells (Magatti et al., 2009; Tipnis et al., 2010; Kronsteiner et al., 2011a,b; Saeidi et al., 2013; Banas et al., 2014; Abomaray et al., 2015; Donders et al., 2015; Magatti et al., 2015), and induce M2 macrophage differentiation (Manuelpillai et al., 2012; Abumaree et al., 2013; Magatti et al., 2015). Moreover, placenta-derived cells have been shown to inhibit neutrophils (Zhou et al., 2003; Li et al., 2005; Chen et al., 2014), and natural killer cells (Ribeiro et al., 2013; Chatterjee et al., 2014; Li et al., 2015).

Even though the underlying mechanisms are not completely understood, there are numerous studies which have put forth different hypotheses. Herein, we will briefly summarize them, since comprehensive reviews of the interactions between placenta-derived and immune cells have been described elsewhere (Parolini et al., 2009; Parolini et al., 2010; Manuelpillai et al., 2011; Parolini and Caruso, 2011; Prasanna and Jahnavi, 2011; Abumaree et al., 2012; Caruso et al., 2012; La Rocca et al., 2012; Kim et al., 2013; Silini et al., 2013; Insausti et al., 2014). Cells from placental tissues have been shown to produce factors shown to dampen inflammation, such as interleukin (IL)-10 (Kronsteiner et al., 2011a,b; Rossi et al., 2012; Abomaray et al., 2015; Magatti et al., 2015), transforming growth factor (TGF)- $\beta$ (Liu et al., 2012; Rossi et al., 2012; Pianta et al., 2015), hepatocyte growth factor (HGF) (Najar et al., 2010; Kronsteiner et al., 2011 a,b; Raicevic et al., 2011; Yamahara et al., 2014), prostaglandin E2 (PGE2), (Whittle et al., 2000; Chen et al., 2010; Najar et al., 2010; Kronsteiner et al., 2011a,b; Raicevic et al., 2011; Liu et al., 2012; Rossi et al., 2012; Liu et al., 2014; Yamahara et al., 2014; Abomaray et al., 2015), and indoleamine 2,3-dioxygenase (IDO) enzyme (Chang et al., 2006; Rossi et al., 2012; Anam et al., 2013; Donders et al., 2015). Moreover, placenta-derived cells express negative co-signaling proteins B7H3, PD-L1 (CD274), and PD-L2 (CD273), (Petroff and Perchellet, 2010; Tipnis et al., 2010; Kronsteiner et al., 2011a,b; La Rocca et al., 2012; Abumaree et al., 2013; Wu et al., 2014). HLA-G, a molecule known to have immune-regulatory properties through its interactions with immunoglobulin-like transcript (ILT) receptors (ILT-2, ILT-3, ILT-4), (Allan et al., 2000; Hunt et al., 2005), has been reported to be secreted by a variety of placenta-derived cells (Lefebvre et al., 2000; Chang et al., 2006; Banas et al., 2008; Roelen et al., 2009; Kronsteiner et al., 2011a,b; Pratama et al., 2011; Anam et al., 2013; Donders et al., 2015).

On another note, inflammatory cytokines/milieu have been shown to enhance the immunomodulatory properties of placenta-derived cells. For example, interferon (IFN)- $\gamma$ has been shown to enhance their anti-proliferative properties on PBMC (Chang et al., 2006; Prasanna et al., 2010; Kronsteiner et al., 2011a,b; Donders et al., 2015). IFN- $\gamma$ has also been shown to increase HLA-G (Lefebvre et al., 2000; Banas et al., 2008; Kronsteiner et al., 2011a,b), PD-L1 and PD-L2 (Banas et al., 2008; Petroff and Perchellet, 2010; Tipnis et al., 2010; Kronsteiner et al., 2011a,b), and PGE2 production by placental cells (Chen et al., 2010). Moreover, IL-1 $\beta$, a potent inflammatory cytokine, has also been shown to enhance the immune modulatory properties of placental cells, such as through the induction of PGE2 secretion (Mitchell et al., 1993; Fukuda et al.,
1999; Pomini et al., 1999; Chen et al., 2010, Phillips et al., 2011), and by enhancing their suppressive activities toward NK cells (Chatterjee et al., 2014).

\section{Pro- and Anti-Angiogenic Properties}

The intact AM has been shown to produce an array of antiangiogenic factors. This property is epitomized in the use of intact AM for corneal surface reconstruction, where the use of AM decreases vascularization of the ocular surface through the production of anti-angiogenic proteins (Kim and Tseng, 1995; Shao et al., 2004), such as pigment epithelium-derived factor (PEDF), (Kim and Tseng, 1995; Dawson et al., 1999; Shao et al., 2004), tissue inhibitor of metalloproteinase (TIMP)-1 and TIMP-2 (Hao et al., 2000), and thrombospondin-1 (TSP-1), (Zaslavsky et al., 2010). The intact AM has also been reported to have a large amount of ECM proteins (i.e., laminin-1, laminin-5, fibronectin), which are involved in the suppression of neovascularization in the cornea (Fukuda et al., 1999).

Decellularized AM, whereby amniotic epithelial cells are eliminated and the cytokine-rich ECM is retained, has been shown to maintain anti-angiogenic properties (Tseng et al., 2004).

Furthermore, epithelial and mesenchymal cells isolated from the AM have also been shown to produce anti-angiogenic factors, such as TIMP-1, TIMP-2, TSP-1, and endostatin (Rowe et al., 1997; Hao et al., 2000).

On the other hand, pro-angiogenic properties have also been attributed to the AM, which can be considered important contributors to its wound healing and regenerative capabilities. For example, cytokines known to promote angiogenesis have been found in dehydrated human amnion/chorion membrane, such as angiopoietin-2, epidermal growth factor (EGF), basic fibroblast growth factor (bFGF), heparin binding epidermal growth factor (HB-EGF), HGF, platelet-derived growth factor BB (PDGF-BB), placental growth factor (PlGF), and VEGF (Koob et al., 2014b). Recently, pro-angiogenic factors have been found in conditioned medium from MSC isolated from the AM, which was shown to not only limit infarct size but also promote capillary formation at the infarct border zone when injected into infarcted rat hearts (Danieli et al., 2015). These apparently contradictory properties could give rise to the importance of understanding the impact of the microenvironment in determining the pro- or anti-angiogenic abilities of the AM and placental cells.

\section{Promotion of Epithelialization}

Another important, and well-documented, property of the intact $\mathrm{AM}$ is its ability to promote re-epithelialization. The intact AM has been used as a basement membrane to promote epithelial cell migration, differentiation, and prevent epithelial cell apoptosis (Dua et al., 2004). In addition, it produces factors that can stimulate epithelialization, such as bFGF, HGF, and TGF $\beta$ (Dua and Azuara-Blanco, 1999; Koizumi et al., 2000). The intact AM also produces factors that support the growth and differentiation of stem and progenitor cells (Meller et al., 2000; Meller et al., 2002, Insausti et al., 2010), such as keratinocyte growth factor (KGF) (Casey and MacDonald, 1997), supporting its use as a progenitor cell niche (Tseng et al., 2004). 
Furthermore, dehydrated human amnion/chorion membrane, which preserves ECM composition and retains an array of cytokines, chemokines, and growth factors naturally present in the native tissue, was found to preserve re-epithelialization properties (Koob et al., 2014a).

Studies performed on the AM denuded of epithelial cells have shown significantly lower levels of bFGF, HGF, EGF, and KGF, when compared to intact AM, suggesting an epithelial origin of these factors (Koizumi et al., 2000). Among other ECM proteins found in AM, fibronectin, laminins, and collagen IV and VII have also been reported, which can in turn promote epithelial adhesion and migration (Fukuda et al., 1999; Lobert et al., 2010).

Cells of the AM have also been suggested to promote epithelialization through secretion/production of factors, which can direct migration, proliferation, and differentiation of keratinocytes. In particular, human amniotic mesenchymal cells (hAMSC) and epithelial cells (hAEC) can secrete factors crucial for wound healing. hAMSC highly express EGF, a factor known for its role in keratinocyte and fibroblast migration, IL-8 that promotes re-epithelialization by increasing keratinocyte proliferation and migration, and IGF-1 that is involved in wound closure by promoting the growth of endothelial cells, dermal fibroblasts, and keratinocytes (Kim et al., 2012).

High expression of EGF and PDGF has also been reported in hAEC, the latter of which stimulates the chemotaxis and proliferation of fibroblasts, and is a critical regulator of ECM deposition in healing wounds (Jin et al., 2015). Both hAMSC and hAEC have been shown to engraft into the wound area thus potentially enhancing their paracrine effects and, furthermore, they could directly participate in re-epithelialization by their trans-differentiation into keratinocytes (Kim et al., 2012; Jin et al., 2015).

\section{REFERENCES}

Abomaray, F. M., Al Jumah, M. A., Kalionis, B., AlAskar, A. S., Al Harthy, S., Jawdat, D., et al. (2015). Human chorionic villous mesenchymal stem cells modify the functions of human dendritic cells, and induce an anti-inflammatory phenotype in CD1+ dendritic cells. Stem Cell. Rev. 11, 423-441. doi:10.1007/ s12015-014-9562-8

Abumaree, M., AlJumah, M., Pace, R.A., and Kalionis, B.(2012).Immunosuppressive properties of mesenchymal stem cells. Stem Cell. Rev. 8, 375-392. doi:10.1007/ s12015-011-9312-0

Abumaree, M. H., Al Jumah, M. A., Kalionis, B., Jawdat, D., Al Khaldi, A., Abomaray, F. M., et al. (2013). Human placental mesenchymal stem cells (pMSCs) play a role as immune suppressive cells by shifting macrophage differentiation from inflammatory M1 to anti-inflammatory M2 macrophages. Stem Cell. Rev. 9, 620-641. doi:10.1007/s12015-013-9455-2

Allan, D. S., McMichael, A. J., and Braud, V. M. (2000). The ILT family of leukocyte receptors. Immunobiology 202, 34-41. doi:10.1016/S0171-2985(00)80050-9

Anam, K., Lazdun, Y., Davis, P. M., Banas, R. A., Elster, E. A., and Davis, T. A. (2013). Amnion-derived multipotent progenitor cells support allograft tolerance induction. Am. J. Transplant. 13, 1416-1428. doi:10.1111/ajt.12252

Arora, M., Jaroudi, K. A., Hamilton, C. J., and Dayel, F. (1994). Controlled comparison of interceed and amniotic membrane graft in the prevention of postoperative adhesions in the rabbit uterine horn model. Eur. J. Obstet. Gynecol. Reprod. Biol. 55, 179-182. doi:10.1016/0028-2243(94)90035-3

Bailo, M., Soncini, M., Vertua, E., Signoroni, P. B., Sanzone, S., Lombardi, G., et al. (2004). Engraftment potential of human amnion and chorion cells

\section{CONCLUDING REMARKS}

Although there are still many open questions regarding the sine qua non conditions for the clinical use of placental cells (Fierabracci et al., 2015), the increasing number of clinical trials underlines the interest in using them. Ongoing and future studies will be crucial in helping define their molecular mechanisms, and establishing the true value of placental derivatives (AM, cells, or molecules they release). These studies will undoubtedly unveil additional applications in the field of regenerative medicine. Studies on placental derivatives available thus far have surely contributed to the vision of regenerative medicine, not only based on cell replacement but also on the importance of their paracrine effects, which could promote endogenous tissue regeneration.

\section{AUTHOR CONTRIBUTIONS}

AS, AC, MM, SP, and OP contributed to writing the manuscript, OP gave final approval of the version to be published. All authors read and approved the manuscript.

\section{ACKNOWLEDGMENTS}

The authors would like to thank Fondazione PoliambulanzaIstituto Ospedaliero of Brescia, and all mothers who donated their baby's placentas for research. This work was supported by Fondazione Poliambulanza-Istituto Ospedaliero, Brescia, Italy, Cariplo Foundation (grant no. 2012-0842), Italian Ministry of Health call "Ricerca Finalizzata" (Project code: RF-20102315681), and Competitiveness ROP ERDF 2007-2013 of Lombardy Region (Regional Operational Programme of the European Regional Development Fund - Progetto NUTEC NUove TECnologie ID no. 30263049).

derived from term placenta. Transplantation 78, 1439-1448. doi:10.1097/01. TP.0000144606.84234.49

Banas, R., Miller, C., Guzik, L., and Zeevi, A. (2014). Amnion-derived multipotent progenitor cells inhibit blood monocyte differentiation into mature dendritic cells. Cell Transplant. 23, 1111-1125. doi:10.3727/096368913X670165

Banas, R. A., Trumpower, C., Bentlejewski, C., Marshall, V., Sing, G., and Zeevi, A. (2008). Immunogenicity and immunomodulatory effects of amnion-derived multipotent progenitor cells. Hum. Immunol. 69, 321-328. doi:10.1016/j. humimm.2008.04.007

Bennett, J. P., Matthews, R., and Faulk, W. P. (1980). Treatment of chronic ulceration of the legs with human amnion. Lancet 1, 1153-1156. doi:10.1016/ S0140-6736(80)91616-5

Bergmann, J., Hackl, F., Koyama, T., Aflaki, P., Smith, C. A., Robson, M. C., et al. (2009). The effect of amnion-derived cellular cytokine solution on the epithelialization of partial-thickness donor site wounds in normal and streptozotocin-induced diabetic swine. Eplasty 9 , e49.

Bose, B. (1979). Burn wound dressing with human amniotic membrane. Ann. R. Coll. Surg. Engl. 61, 444-447.

Brindeau, A. (1934). Creation d'un vagin artificiel A l'aide des membranes ovulaires d'un oeuf A terme. J. Gynecol. Obstet. Biol. Reprod (Paris) 13, 821-824.

Brooke, G., Rossetti, T., Pelekanos, R., Ilic, N., Murray, P., Hancock, S., et al. (2009). Manufacturing of human placenta-derived mesenchymal stem cells for clinical trials. Br. J. Haematol. 144, 571-579. doi:10.1111/j.1365-2141.2008.07492.x

Burger, K. (1937). Weitere erfahrungen iiber die kunstliche scheidenbildung mit eihauten. Zentralbl. Gynäkol. 69, 1153-1154. 
Caruso, M., Evangelista, M., and Parolini, O. (2012). Human term placental cells: phenotype, properties and new avenues in regenerative medicine. Int. J. Mol. Cell Med. 1, 64-74.

Casey, M. L., and MacDonald, P. C. (1997). Keratinocyte growth factor expression in the mesenchymal cells of human amnion. J. Clin. Endocrinol. Metab. 82, 3319-3323. doi:10.1210/jcem.82.1.3668

Chambers, D. C., Enever, D., Ilic, N., Sparks, L., Whitelaw, K., Ayres, J., et al. (2014). A phase $1 \mathrm{~b}$ study of placenta-derived mesenchymal stromal cells in patients with idiopathic pulmonary fibrosis. Respirology 19, 1013-1018. doi:10.1111/ resp. 12343

Chang, C. J., Yen, M. L., Chen, Y. C., Chien, C. C., Huang, H. I., Bai, C. H., et al. (2006). Placenta-derived multipotent cells exhibit immunosuppressive properties that are enhanced in the presence of interferon-gamma. Stem Cells 24, 2466-2477. doi:10.1634/stemcells.2004-0308

Chao, Y.-C., Humphreys, S., and Penfield, W. (1940). A new method of preventing adhesions. The use of amnioplastin after craniotomy. Br. Med. J. 1, 517. doi:10.1136/bmj.1.4134.517

Chatterjee, D., Marquardt, N., Tufa, D. M., Beauclair, G., Low, H. Z., Hatlapatka, T., et al. (2014). Role of gamma-secretase in human umbilical-cord derived mesenchymal stem cell mediated suppression of NK cell cytotoxicity. Cell Commun. Signal. 12, 63. doi:10.1186/s12964-014-0063-9

Chen, C. P., Chen, Y. Y., Huang, J. P., and Wu, Y. H. (2014). The effect of conditioned medium derived from human placental multipotent mesenchymal stromal cells on neutrophils: possible implications for placental infection. Mol. Hum. Reprod. 20, 1117-1125. doi:10.1093/molehr/gau062

Chen, K., Wang, D., Du, W. T., Han, Z. B., Ren, H., Chi, Y., et al. (2010). Human umbilical cord mesenchymal stem cells hUC-MSCs exert immunosuppressive activities through a PGE2-dependent mechanism. Clin. Immunol. 135, 448-458. doi:10.1016/j.clim.2010.01.015

Danieli, P., Malpasso, G., Ciuffreda, M. C., Cervio, E., Calvillo, L., Copes, F., et al. (2015). Conditioned medium from human amniotic mesenchymal stromal cells limits infarct size and enhances angiogenesis. Stem Cells Transl. Med. 4, 448-458. doi:10.5966/sctm.2014-0253

Davis, J. (1910). Skin transplantation with a review of 550 cases at the Johns Hopkins hospital. Johns Hopkins Med. J. 15, 15.

Dawson, D. W., Volpert, O. V., Gillis, P., Crawford, S. E., Xu, H., Benedict, W., et al. (1999). Pigment epithelium-derived factor: a potent inhibitor of angiogenesis. Science 285, 245-248. doi:10.1126/science.285.5425.245

de Farias, C. C., Sterlenich, T., de Sousa, L. B., Vieira, L. A., and Gomes, J. A. (2014). Randomized trial comparing multilayer amniotic membrane transplantation with scleral and corneal grafts for the treatment of scleral thinning after pterygium surgery associated with beta therapy. Cornea 33, 1197-1204. doi:10.1097/ ICO.0000000000000207

De Rotth, A. (1940). Plastic repair of conjunctival defects with fetal membranes. Arch. Ophthalmol. 23, 522. doi:10.1097/ICO.0b013e318156cb08

Dhall, K. (1984). Amnion graft for treatment of congenital absence of the vagina. Br. J. Obstet. Gynaecol. 91, 279-282. doi:10.1111/j.1471-0528.1984. tb04768.x

Dino, B., Eufemio, G., De Villa, M., Reysio-Cruz, M., and Jurado, R. (1965). The use of fetal membrane homografts in the local management of burns. J. Philipp. Med. Assoc. 41, 890.

Donders, R., Vanheusden, M., Bogie, J. F., Ravanidis, S., Thewissen, K., Stinissen, P., et al. (2015). Human Wharton's jelly-derived stem cells display immunomodulatory properties and transiently improve rat experimental autoimmune encephalomyelitis. Cell Transplant. 24, 2077-2098. doi:10.3727/096368914X685104

Douglas, B., Conway, H., Stark, R. B., Joslin, D., and Nieto-Cano, G. (1954). The fate of homologous and heterologous chorionic transplants as observed by the transparent tissue chamber technique in the mouse. Plast. Reconstr. Surg. 13, 125-129. doi:10.1097/00006534-195402000-00005

Dua, H. S., and Azuara-Blanco, A. (1999). Amniotic membrane transplantation. Br. J. Ophthalmol. 83, 748-752. doi:10.1136/bjo.83.7.885a

Dua, H. S., Gomes, J. A., King, A. J., and Maharajan, V. S. (2004). The amniotic membrane in ophthalmology. Surv. Ophthalmol. 49, 51-77. doi:10.1016/j. survophthal.2003.10.004

Erkers, T., Kaipe, H., Nava, S., Mollden, P., Gustafsson, B., Axelsson, R., et al. (2015). Treatment of severe chronic graft-versus-host disease with decidual stromal cells and tracing with (111)indium radiolabeling. Stem Cells Dev. 24, 253-263. doi: $10.1089 /$ scd.2014.0265
Fetterolf, D. E., and Snyder, R. J. (2012). Scientific and clinical support for the use of dehydrated amniotic membrane in wound management. Wounds 24, 299-307.

Fierabracci, A., Lazzari, L., Muraca, M., and Parolini, O. (2015). How far are we from the clinical use of placental-derived mesenchymal stem cells? Expert Opin. Biol. Ther. 15, 613-617. doi:10.1517/14712598.2015.1000856

Fukuchi, Y., Nakajima, H., Sugiyama, D., Hirose, I., Kitamura, T., and Tsuji, K. (2004). Human placenta-derived cells have mesenchymal stem/progenitor cell potential. Stem Cells 22, 649-658. doi:10.1634/stemcells.22-5-649

Fukuda, K., Chikama, T., Nakamura, M., and Nishida, T. (1999). Differential distribution of subchains of the basement membrane components type IV collagen and laminin among the amniotic membrane, cornea, and conjunctiva. Cornea 18, 73-79. doi:10.1097/00003226-199901000-00013

Georgy, M., and Aziz, N. (1996). Vaginoplasty using amnion graft: new surgical technique using the laparoscopic transillumination light. J. Obstet. Gynaecol. 16, 262-264. doi:10.3109/01443619609020728

Gharib, M., Ure, B. M., and Klose, M. (1996). Use of amniotic grafts in the repair of gastroschisis. Pediatr. Surg. Int. 11, 96-99. doi:10.1007/BF00183734

Gruss, J. S., and Jirsch, D. W. (1978). Human amniotic membrane: a versatile wound dressing. Can. Med. Assoc. J. 118, 1237-1246.

Hao, Y., Ma, D. H., Hwang, D. G., Kim, W. S., and Zhang, F. (2000). Identification of antiangiogenic and antiinflammatory proteins in human amniotic membrane. Cornea 19, 348-352. doi:10.1097/00003226-200005000-00018

Hunt, J. S., Petroff, M. G., McIntire, R. H., and Ober, C. (2005). HLA-G and immune tolerance in pregnancy. FASEB J. 19, 681-693. doi:10.1096/fj.04-2078rev

Igura, K., Zhang, X., Takahashi, K., Mitsuru, A., Yamaguchi, S., and Takashi, T. A. (2004). Isolation and characterization of mesenchymal progenitor cells from chorionic villi of human placenta. Cytotherapy 6, 543-553. doi:10.1080/14653240410005366-1

In 't Anker, P. S., Scherjon, S. A., Kleijburg-van der Keur, C., de Groot-Swings, G. M., Claas, F. H., Fibbe, W. E., et al. (2004). Isolation of mesenchymal stem cells of fetal or maternal origin from human placenta. Stem Cells 22, 1338-1345. doi:10.1634/stemcells.2004-0058

Insausti, C. L., Alcaraz, A., Garcia-Vizcaino, E. M., Mrowiec, A., Lopez-Martinez, M. C., Blanquer, M., et al. (2010). Amniotic membrane induces epithelialization in massive posttraumatic wounds. Wound Repair Regen. 18, 368-377. doi:10.1111/j.1524-475X.2010.00604.X

Insausti, C. L., Blanquer, M., Garcia-Hernandez, A. M., Castellanos, G., and Moraleda, J. M. (2014). Amniotic membrane-derived stem cells: immunomodulatory properties and potential clinical application. Stem Cells Cloning 7, 53-63. doi:10.2147/SCCAA.S58696

Jiang, R., Han, Z., Zhuo, G., Qu, X., Li, X., Wang, X., et al. (2011). Transplantation of placenta-derived mesenchymal stem cells in type 2 diabetes: a pilot study. Front Med. 5:94-100. doi:10.1007/s11684-011-0116-Z

Jin, E., Kim, T. H., Han, S., and Kim, S. W. (2015). Amniotic epithelial cells promote wound healing in mice through high epithelialization and engraftment. J. Tissue Eng. Regen. Med. doi:10.1002/term.2069

Kaipe, H., Carlson, L. M., Erkers, T., Nava, S., Mollden, P., Gustafsson, B., et al. (2015). Immunogenicity of decidual stromal cells in an epidermolysis bullosa patient and in allogeneic hematopoietic stem cell transplantation patients. Stem Cells Dev. 24, 1471-1482. doi:10.1089/scd.2014.0568

Kang, L., Voskinarian-Berse, V., Law, E., Reddin, T., Bhatia, M., Hariri, A., et al. (2013). Characterization and ex vivo expansion of human placenta-derived natural killer cells for cancer immunotherapy. Front. Immunol. 4:101. doi:10.3389/ fimmu.2013.00101

Kesting, M. R., Wolff, K. D., Nobis, C. P., and Rohleder, N. H. (2014). Amniotic membrane in oral and maxillofacial surgery. Oral Maxillofac. Surg. 18, 153-164. doi:10.1007/s10006-012-0382-1

Kim, D. W., Staples, M., Shinozuka, K., Pantcheva, P., Kang, S. D., and Borlongan, C. V. (2013). Wharton's jelly-derived mesenchymal stem cells: phenotypic characterization and optimizing their therapeutic potential for clinical applications. Int. J. Mol. Sci. 14, 11692-11712. doi:10.3390/ijms140611692

Kim, J. C., and Tseng, S. C. (1995). The effects on inhibition of corneal neovascularization after human amniotic membrane transplantation in severely damaged rabbit corneas. Korean J. Ophthalmol. 9, 32-46. doi:10.3341/kjo.1995.9.1.12

Kim, S. W., Zhang, H. Z., Guo, L., Kim, J. M., and Kim, M. H. (2012). Amniotic mesenchymal stem cells enhance wound healing in diabetic NOD/SCID mice through high angiogenic and engraftment capabilities. PLOS ONE 7:e41105. doi:10.1371/journal.pone.0041105 
Koizumi, N. J., Inatomi, T. J., Sotozono, C. J., Fullwood, N. J., Quantock, A. J., and Kinoshita, S. (2000). Growth factor mRNA and protein in preserved human amniotic membrane. Curr. Eye Res. 20, 173-177. doi:10.1076/0271-3683(200003)2031-9FT173

Koob, T. J., Lim, J. J., Massee, M., Zabek, N., and Denoziere, G. (2014a). Properties of dehydrated human amnion/chorion composite grafts: Implications for wound repair and soft tissue regeneration. J. Biomed. Mater. Res. Part B Appl. Biomater. 102, 1353-1362. doi:10.1002/jbm.b.33141

Koob, T. J., Lim, J. J., Massee, M., Zabek, N., Rennert, R., Gurtner, G., et al. (2014b). Angiogenic properties of dehydrated human amnion/chorion allografts: therapeutic potential for soft tissue repair and regeneration. Vasc. Cell 6, 10. doi:10.1186/2045-824X-6-10

Kordić, R., Suić, S. P., Jandroković, S., Kalauz, M., Kuzman, T., Skegro, I., et al. (2013). Application of the amniotic membrane extract (AMX) for the persistent epithelial defect (PED) of the cornea. Coll. Antropol. 37(Suppl. 1), 161-164.

Kranz, A., Wagner, D. C., Kamprad, M., Scholz, M., Schmidt, U. R., Nitzsche, F., et al. (2010). Transplantation of placenta-derived mesenchymal stromal cells upon experimental stroke in rats. Brain Res. 1315, 128-136. doi:10.1016/j. brainres.2009.12.001

Kronsteiner, B., Peterbauer-Scherb, A., Grillari-Voglauer, R., Redl, H., Gabriel, C., van Griensven, M., et al. (2011a). Human mesenchymal stem cells and renal tubular epithelial cells differentially influence monocyte-derived dendritic cell differentiation and maturation. Cell. Immunol. 267, 30-38. doi:10.1016/j. cellimm.2010.11.001

Kronsteiner, B., Wolbank, S., Peterbauer, A., Hackl, C., Redl, H., Griensven, M. V., et al. (2011b). Human mesenchymal stem cells from adipose tissue and amnion influence T-cells depending on stimulation method and presence of other immune cells. Stem Cells Dev. 20, 2115-2126. doi:10.1089/ scd.2011.0031

Kubanyi, A. (1947). Prevention of peritoneal adhesions by transplantation of amnion. Br. Med. J. 2, 55. doi:10.1136/bmj.2.4514.55-a

La Rocca, G., Corrao, S., Lo Iacono, M., Corsello, T., Farina, F., and Anzalone, R. (2012). Novel immunomodulatory markers expressed by human WJ-MSC: an updated review in regenerative and reparative medicine. Open Tissue Eng. Regen. Med. J. 5, 50-58. doi:10.2174/1875043501205010050

Lee, S.-H., and Tseng, S. C. (1997). Amniotic membrane transplantation for persistent epithelial defects with ulceration. Am. J. Ophthalmol. 123, 303-312. doi:10.1016/S0002-9394(14)70125-4

Lefebvre, S., Adrian, F., Moreau, P., Gourand, L., Dausset, J., Berrih-Aknin, S., et al. (2000). Modulation of HLA-G expression in human thymic and amniotic epithelial cells. Hum. Immunol. 61, 1095-1101. doi:10.1016/S0198-8859(00)00192-0

Li, H., Niederkorn, J. Y., Neelam, S., Mayhew, E., Word, R. A., McCulley, J. P., et al. (2005). Immunosuppressive factors secreted by human amniotic epithelial cells. Invest. Ophthalmol. Vis. Sci. 46, 900-907. doi:10.1167/iovs.05-0034

Li, J., Koike-Soko, C., Sugimoto, J., Yoshida, T., Okabe, M., and Nikaido, T. (2015). Human amnion-derived stem cells have immunosuppressive properties on NK cells and monocytes. Cell Transplant. 24, 2065-2076. doi:10.3727/09636 8914 X685230

Liu, J., Sheha, H., Fu, Y., Liang, L., and Tseng, S. C. (2010). Update on amniotic membrane transplantation. Expert Rev. Ophthalmol. 5, 645-661. doi:10.1586/ eop. 10.63

Liu, W., Morschauser, A., Zhang, X., Lu, X., Gleason, J., He, S., et al. (2014). Human placenta-derived adherent cells induce tolerogenic immune responses. Clin. Transl. Immunology 3, e14. doi:10.1038/cti.2014.5

Liu, Y. H., Vaghjiani, V., Tee, J. Y., To, K., Cui, P., Oh, D. Y., et al. (2012). Amniotic epithelial cells from the human placenta potently suppress a mouse model of multiple sclerosis. PLoS ONE 7:e35758. doi:10.1371/journal.pone.0035758

Lo, V., and Pope, E. (2009). Amniotic membrane use in dermatology. Int. J. Dermatol. 48, 935-940. doi:10.1111/j.1365-4632.2009.04173.x

Lobert, V. H., Brech, A., Pedersen, N. M., Wesche, J., Oppelt, A., Malerod, L., et al. (2010). Ubiquitination of alpha 5 beta 1 integrin controls fibroblast migration through lysosomal degradation of fibronectin-integrin complexes. Dev. Cell 19, 148-159. doi:10.1016/j.devcel.2010.06.010

Lublin, F. D., Bowen, J. D., Huddlestone, J., Kremenchutzky, M., Carpenter, A., Corboy, J. R., et al. (2014). Human placenta-derived cells (PDA-001) for the treatment of adults with multiple sclerosis: a randomized, placebo-controlled, multiple-dose study. Mult. Scler. Relat. Disord. 3, 696-704. doi:10.1016/j. msard.2014.08.002
Ma, L., Zhou, Z., Zhang, D., Yang, S., Wang, J., Xue, F., et al. (2012). Immunosuppressive function of mesenchymal stem cells from human umbilical cord matrix in immune thrombocytopenia patients. Thromb. Haemost. 107, 937-950. doi:10.1160/TH11-08-0596

Magatti, M., Caruso, M., De Munari, S., Vertua, E., De, D., Manuelpillai, U., et al. (2015). Human amniotic membrane-derived mesenchymal and epithelial cells exert different effects on monocyte-derived dendritic cell differentiation and function. Cell Transplant. 24, 1733-1752. doi:10.3727/096368914X684033

Magatti, M., De Munari, S., Vertua, E., Nassauto, C., Albertini, A., Wengler, G. S., et al. (2009). Amniotic mesenchymal tissue cells inhibit dendritic cell differentiation of peripheral blood and amnion resident monocytes. Cell Transplant. 18, 899-914. doi:10.3727/096368909X471314

Manuelpillai, U., Lourensz, D., Vaghjiani, V., Tchongue, J., Lacey, D., Tee, J. Y., et al. (2012). Human amniotic epithelial cell transplantation induces markers of alternative macrophage activation and reduces established hepatic fibrosis. PLoS ONE 7:e38631. doi:10.1371/journal.pone.0038631

Manuelpillai, U., Moodley, Y., Borlongan, C. V., and Parolini, O. (2011). Amniotic membrane and amniotic cells: potential therapeutic tools to combat tissue inflammation and fibrosis? Placenta 32(Suppl. 4), S320-S325. doi:10.1016/j. placenta.2011.04.010

Mayer, L., Pandak, W. M., Melmed, G. Y., Hanauer, S. B., Johnson, K., Payne, D., et al. (2013). Safety and tolerability of human placenta-derived cells (PDA001) in treatment-resistant Crohn's disease: a phase I study. Inflamm. Bowel Dis. 19, 754-760. doi:10.1097/MIB.0b013e31827f27df

McIntyre, J., and Faulk, W. P. (1979). Antigens of human trophoblast. Effects of heterologous anti-trophoblast sera on lymphocyte responses in vitro. J. Exp. Med. 149, 824-836. doi:10.1084/jem.149.4.824

Meller, D., Pires, R. T., Mack, R. J., Figueiredo, F., Heiligenhaus, A., Park, W. C., et al. (2000). Amniotic membrane transplantation for acute chemical or thermal burns. Ophthalmology 107, 980-989. doi:10.1016/S0161-6420(00)00024-5

Meller, D., Pires, R. T., and Tseng, S. C. (2002). Ex vivo preservation and expansion of human limbal epithelial stem cells on amniotic membrane cultures. $\mathrm{Br}$. J. Ophthalmol. 86, 463-471. doi:10.1136/bjo.86.4.463

Min, S., Yoon, J. Y., Park, S. Y., Kwon, H. H., and Suh, D. H. (2014). Clinical effect of bovine amniotic membrane and hydrocolloid on wound by laser treatment: prospective comparative randomized clinical trial. Wound Repair Regen. 22, 212-219. doi:10.1111/wrr.12145

Mitchell, M. D., Edwin, S. S., Lundin-Schiller, S., Silver, R. M., Smotkin, D., and Trautman, M. S. (1993). Mechanism of interleukin-1 beta stimulation of human amnion prostaglandin biosynthesis: mediation via a novel inducible cyclooxygenase. Placenta 14, 615-625. doi:10.1016/S0143-4004(05)80426-6

Muralidharan, S., Gu, J., and Laub, G. (1991). A new biological membrane for pericardial closure. J. Biomed. Mater. Res. 25, 1201-1209. doi:10.1002/ jbm.820251003

Najar, M., Raicevic, G., Boufker, H. I., Fayyad Kazan, H., De Bruyn, C., Meuleman, N., et al. (2010). Mesenchymal stromal cells use PGE2 to modulate activation and proliferation of lymphocyte subsets: combined comparison of adipose tissue, Wharton's jelly and bone marrow sources. Cell. Immunol. 264, 171-179. doi:10.1016/j.cellimm.2010.06.006

Nisolle, M., and Donnez, J. (1992). Vaginoplasty using amniotic membranes in cases of vaginal agenesis or after vaginectomy. J. Gynaecol. Surg. 8, 25-30. doi:10.1089/gyn.1992.8.25

Ohshima, M., Yamahara, K., Ishikane, S., Harada, K., Tsuda, H., Otani, K., et al. (2012). Systemic transplantation of allogenic fetal membrane-derived mesenchymal stem cells suppresses Th1 and Th17 T cell responses in experimental autoimmune myocarditis. J. Mol. Cell. Cardiol. 53, 420-428. doi:10.1016/j. yjmcc.2012.06.020

Paris Fdos, S., Goncalves, E. D., Campos, M. S., Sato, E. H., Dua, H. S., and Gomes, J. A. (2013). Amniotic membrane transplantation versus anterior stromal puncture in bullous keratopathy: a comparative study. Br. J. Ophthalmol. 97, 980-984. doi:10.1136/bjophthalmol-2013-303081

Parolini, O., Alviano, F., Bergwerf, I., Boraschi, D., De Bari, C, De Waele, P, et al. (2010). Toward cell therapy using placenta-derived cells: disease mechanisms, cell biology, preclinical studies, and regulatory aspects at the round table. Stem Cells Dev. 19, 143-154. doi:10.1089/scd.2009.0404

Parolini, O., and Caruso, M. (2011). Review: preclinical studies on placenta-derived cells and amniotic membrane: an update. Placenta 32(Suppl. 2), S186-S195. doi:10.1016/j.placenta.2010.12.016 
Parolini, O., Soncini, M., Evangelista, M., and Schmidt, D. (2009). Amniotic membrane and amniotic fluid-derived cells: potential tools for regenerative medicine? Regen. Med. 4, 275-291. doi:10.2217/17460751.4.2.275

Parolini, O., Souza-Moreira, L., O’Valle, F., Magatti, M., Hernandez-Cortes, P., Gonzalez-Rey, E., et al. (2014). Therapeutic effect of human amniotic membrane-derived cells on experimental arthritis and other inflammatory disorders. Arthritis Rheumatol. 66, 327-339. doi:10.1002/art.38206

Petroff, M. G., and Perchellet, A. (2010). B7 family molecules as regulators of the maternal immune system in pregnancy. Am. J. Reprod. Immunol. 63, 506-519. doi:10.1111/j.1600-0897.2010.00841.x

Phillips, R. J., Al-Zamil, H., Hunt, L. P., Fortier, M. A., and ópez Bernal, A. L. (2011). Genes for prostaglandin synthesis, transport and inactivation are differentially expressed in human uterine tissues, and the prostaglandin F synthase AKR1B1 is induced in myometrial cells by inflammatory cytokines. Mol. Hum. Reprod. 17, 1-13. doi:10.1093/molehr/gaq057

Pianta, S., Bonassi Signoroni, P., Muradore, I., Rodrigues, M. F., Rossi, D., Silini, A., et al. (2015). Amniotic membrane mesenchymal cells-derived factors skew $\mathrm{T}$ cell polarization toward treg and downregulate Th1 and Th17 cells subsets. Stem Cell. Rev. 11, 394-407. doi:10.1007/s12015-014-9558-4

Pomini, F., Caruso, A., and Challis, J. R. (1999). Interleukin-10 modifies the effects of interleukin-1beta and tumor necrosis factor-alpha on the activity and expression of prostaglandin $\mathrm{H}$ synthase-2 and the NAD+-dependent 15-hydroxyprostaglandin dehydrogenase in cultured term human villous trophoblast and chorion trophoblast cells. J. Clin. Endocrinol. Metab. 84, 4645-4651. doi:10.1210/jcem.84.12.6188

Prasanna, S., and Jahnavi, V. (2011). Wharton's jelly mesenchymal stem cells as off-the-shelf cellular therapeutics: a closer look into their regenerative and immunomodulatory properties. Open Tissue Eng. Regen. Med. J. 2011, 28-38. d oi:10.2174/1875043501104010028

Prasanna, S. J., Gopalakrishnan, D., Shankar, S. R., and Vasandan, A. B. (2010). Pro-inflammatory cytokines, IFNgamma and TNFalpha, influence immune properties of human bone marrow and Wharton jelly mesenchymal stem cells differentially. PLoS ONE 5:e9016. doi:10.1371/journal.pone.0009016

Pratama, G., Vaghjiani, V., Tee, J. Y., Liu, Y. H., Chan, J., Tan, C., et al. (2011). Changes in culture expanded human amniotic epithelial cells: implications for potential therapeutic applications. PLoS ONE 6:e26136. doi:10.1371/journal. pone.0026136

Raicevic, G., Najar, M., Stamatopoulos, B., De Bruyn, C., Meuleman, N., Bron, D., et al. (2011). The source of human mesenchymal stromal cells influences their TLR profile as well as their functional properties. Cell. Immunol. 270, 207-216. doi:10.1016/j.cellimm.2011.05.010

Ramot, Y., Meiron, M., Toren, A., Steiner, M., and Nyska, A. (2009). Safety and biodistribution profile of placental-derived mesenchymal stromal cells (PLX-PAD) following intramuscular delivery. Toxicol. Pathol. 37, 606-616. doi: $10.1177 / 0192623309338383$

Rennekampff, H. O., Dohrmann, P., Fory, R., and Fandrich, F. (1994). Evaluation of amniotic membrane as adhesion prophylaxis in a novel surgical gastroschisis model. J. Invest. Surg. 7, 187-193. doi:10.3109/08941939409018285

Riau, A. K., Beuerman, R. W., Lim, L. S., and Mehta, J. S. (2010). Preservation, sterilization and de-epithelialization of human amniotic membrane for use in ocular surface reconstruction. Biomaterials 31, 216-225. doi:10.1016/j. biomaterials.2009.09.034

Ribeiro, A., Laranjeira, P., Mendes, S., Velada, I., Leite, C., Andrade, P., et al. (2013). Mesenchymal stem cells from umbilical cord matrix, adipose tissue and bone marrow exhibit different capability to suppress peripheral blood B, natural killer and T cells. Stem Cell Res. Ther. 4, 125. doi:10.1186/scrt336

Ringden, O., Erkers, T., Nava, S., Uzunel, M., Iwarsson, E., Conrad, R., et al. (2013). Fetal membrane cells for treatment of steroid-refractory acute graft-versus-host disease. Stem Cells 31, 592-601. doi:10.1002/stem.1314

Roelen, D. L., van der Mast, B. J., in 't Anker, P. S., Kleijburg, C., Eikmans, M., van Beelen, E., et al. (2009). Differential immunomodulatory effects of fetal versus maternal multipotent stromal cells. Hum. Immunol. 70, 16-23. doi:10.1016/j. humimm.2008.10.016

Rossi, D., Pianta, S., Magatti, M., Sedlmayr, P., and Parolini, O. (2012). Characterization of the conditioned medium from amniotic membrane cells: prostaglandins as key effectors of its immunomodulatory activity. PLOS ONE 7:e46956. doi:10.1371/journal.pone.0046956

Rowe, T. F., King, L. A., MacDonald, P. C., and Casey, M. L. (1997). Tissue inhibitor of metalloproteinase- 1 and tissue inhibitor of metalloproteinase-2 expression in human amnion mesenchymal and epithelial cells. Am. J. Obstet. Gynecol. 176, 915-921. doi:10.1016/S0002-9378(97)70621-5

Sabella, N. (1913). Use of fetal membranes in skin grafting. Med. Rec. 83, 478-480.

Saeidi, M., Masoud, A., Shakiba, Y., Hadjati, J., Mohyeddin Bonab, M., Nicknam, M. H., et al. (2013). Immunomodulatory effects of human umbilical cord Wharton's jelly-derived mesenchymal stem cells on differentiation, maturation and endocytosis of monocyte-derived dendritic cells. Iran. J. Allergy Asthma Immunol. 12, 37-49. doi:012.01/ijaai.3749

Serena, T. E., Carter, M. J., Le, L. T., Sabo, M. J., DiMarco, D. T., and Group, E. V. S. (2014). A multicenter, randomized, controlled clinical trial evaluating the use of dehydrated human amnion/chorion membrane allografts and multilayer compression therapy vs. multilayer compression therapy alone in the treatment of venous leg ulcers. Wound Repair Regen. 22, 688-693. doi:10.1111/wrr.12227

Shao, C., Sima, J., Zhang, S. X., Jin, J., Reinach, P., Wang, Z., et al. (2004). Suppression of corneal neovascularization by PEDF release from human amniotic membranes. Invest. Ophthalmol. Vis. Sci. 45, 1758-1762. doi:10.1167/iovs.04-0554

Silini, A., Parolini, O., Huppertz, B., and Lang, I. (2013). Soluble factors of amnion-derived cells in treatment of inflammatory and fibrotic pathologies. Curr. Stem Cell Res. Ther. 8, 6-14. doi:10.2174/1574888X11308010003

Silverton, J., Trelford, J., and Roussere, J. (1979). The use of amniotic membrane in acute massive full-thickness loss of the abdominal wall from clostridial myonecrosis. Ann. Plast. Surg. 3, 558-566. doi:10.1097/00000637-197912000-00012

Soncini, M., Vertua, E., Gibelli, L., Zorzi, F., Denegri, M., Albertini, A., et al. (2007). Isolation and characterization of mesenchymal cells from human fetal membranes. J. Tissue Eng. Regen. Med. 1, 296-305. doi:10.1002/term.40

Sorsby, A., Haythorne, J., and Reed, H. (1947). Further experience with amniotic membrane grafts in caustic burns of the eye. Br. J. Ophthalmol. 31, 409-418. doi:10.1136/bjo.31.7.409

Sorsby, A., and Symons, H. M. (1946). Amniotic membrane grafts in caustic burns of the eye (burns of the second degree). Br. J. Ophthalmol. 30, 337-345. doi:10.1136/bjo.30.1.64

Steed, D. L., Trumpower, C., Duffy, D., Smith, C., Marshall, V., Rupp, R., et al. (2008). Amnion-derived cellular cytokine solution: a physiological combination of cytokines for wound healing. Eplasty 8, e18.

Stern, M. (1913). The grafting of preserved amniotic membranes to burned and ulcerated surfaces, substituting skin grafts. JAMA 60, 973. doi:10.1001/ jama.1913.04340130021008

Subrahmanyam, M. (1995). Amniotic membrane as a cover for microskin grafts. Br. J. Plast. Surg. 48, 477-478. doi:10.1016/0007-1226(95)90123-X

Tipnis, S., Viswanathan, C., and Majumdar, A. S. (2010). Immunosuppressive properties of human umbilical cord-derived mesenchymal stem cells: role of B7-H1 and IDO. Immunol. Cell Biol. 88, 795-806. doi:10.1038/icb.2010.47

Trelford, J., Anderson, D., Hanson, F., Mendel, V., and Sawyer, R. (1972). Amnion autografts and allografts as a cover for skin defects in sheep. A preliminary report. J. Med. 3, 81 .

Trelford, J., Hanson, F., Anderson, D., and Mendel, V. (1974). Implanted amniotic membrane as an autograft and as an allograft. J. Med. 6, 169-180.

Trelford-Sauder, M., Dawe, E. J., and Trelford, J. D. (1978). Use of allograft amniotic membrane for control of intra-abdominal adhesions. J. Med. 9, 273-284.

Trelford-Sauder, M., Trelford, J., and Matolo, N. (1977). Replacement of the peritoneum with amnion following pelvic exenteration. Surg. Gynecol. Obstet. $145,699-701$.

Troensagaard-Hansen, E. (1950). Amniotic grafts in chronic skin ulceration. Lancet 1, 859-860. doi:10.1016/S0140-6736(50)90693-3

Troyer, D. L., and Weiss, M. L. (2008). Wharton's jelly-derived cells are a primitive stromal cell population. Stem Cells 26, 591-599. doi:10.1634/ stemcells.2007-0439

Tseng, S. C., Espana, E. M., Kawakita, T., Di Pascuale, M. A., Li, W., He, H., et al. (2004). How does amniotic membrane work? Ocul. Surf. 2, 177-187. doi:10.1016/S1542-0124(12)70059-9

Tseng, S. C., Prabhasawat, P., and Lee, S.-H. (1997). Amniotic membrane transplantation for conjunctival surface reconstruction. Am. J. Ophthalmol. 124, 765-774. doi:10.1016/S0002-9394(14)71693-9

Venegas, L., Hettich, M., Villena, J., Aris, R., Párraga, M., Parolini, O., et al. (2014). Comparative analysis of human amniotic membrane graft versus contact lenses in symptomatic bullous keratopathy. J. Stem Cell Res. Ther. 4, 250. doi:10.4172/2157-7633.1000250

Whittle, W. L., Gibb, W., and Challis, J. R. (2000). The characterization of human amnion epithelial and mesenchymal cells: the cellular expression, activity 
and glucocorticoid regulation of prostaglandin output. Placenta 21, 394-401. doi:10.1053/plac.1999.0482

Wolbank, S., Peterbauer, A., Fahrner, M., Hennerbichler, S., van Griensven, M., Stadler, G., et al. (2007). Dose-dependent immunomodulatory effect of human stem cells from amniotic membrane: a comparison with human mesenchymal stem cells from adipose tissue. Tissue Eng. 13, 1173-1183. doi:10.1089/ ten.2006.0313

Wu, W., Lan, Q., Lu, H., Xu, J., Zhu, A., Fang, W., et al. (2014). Human amnion mesenchymal cells negative co-stimulatory molecules PD-L1 expression and its capacity of modulating microglial activation of CNS. Cell Biochem. Biophys. 69, 35-45. doi:10.1007/s12013-013-9763-9

Yamahara, K., Harada, K., Ohshima, M., Ishikane, S., Ohnishi, S., Tsuda, H., et al. (2014). Comparison of angiogenic, cytoprotective, and immunosuppressive properties of human amnion- and chorion-derived mesenchymal stem cells. PLoS ONE 9:e88319. doi:10.1371/journal.pone.0088319

Young, R. L., Cota, J., Zund, G., Mason, B. A., and Wheeler, J. M. (1991). The use of an amniotic membrane graft to prevent postoperative adhesions. Fertil. Steril. $55,624-628$.

Young, S. M., and Benyshek, D. C. (2010). In search of human placentophagy: a cross-cultural survey of human placenta consumption, disposal practices, and cultural beliefs. Ecol. Food Nutr. 49, 467-484. doi:10.1080/03670244.201 0.524106

Zaslavsky, A., Baek, K. H., Lynch, R. C., Short, S., Grillo, J., Folkman, J., et al. (2010). Platelet-derived thrombospondin-1 is a critical negative regulator and potential biomarker of angiogenesis. Blood 115, 4605-4613. doi:10.1182/ blood-2009-09-242065

Zelen, C. M., Poka, A., and Andrews, J. (2013). Prospective, randomized, blinded, comparative study of injectable micronized dehydrated amniotic/chorionic membrane allograft for plantar fasciitis - a feasibility study. Foot Ankle Int. 34, 1332-1339. doi:10.1177/1071100713502179

Zelen, C. M., Serena, T. E., and Snyder, R. J. (2014). A prospective, randomised comparative study of weekly versus biweekly application of dehydrated human amnion/chorion membrane allograft in the management of diabetic foot ulcers. Int. Wound J. 11, 122-128. doi:10.1111/iwj.12242

Zhou, S., Chen, J., and Feng, J. (2003). The effects of amniotic membrane on polymorphonuclear cells. Chin. Med. J. (Engl). 116, 788-790.

Conflict of Interest Statement: The authors declare that the research was conducted in the absence of any commercial or financial relationships that could be construed as a potential conflict of interest.

Copyright $\odot 2015$ Silini, Cargnoni, Magatti, Pianta and Parolini. This is an open-access article distributed under the terms of the Creative Commons Attribution License (CC BY). The use, distribution or reproduction in other forums is permitted, provided the original author(s) or licensor are credited and that the original publication in this journal is cited, in accordance with accepted academic practice. No use, distribution or reproduction is permitted which does not comply with these terms. 